\title{
Deleterious effects of endocrine disruptors are corrected in the mammalian germline by epigenome reprogramming
}

Khursheed lqbal ${ }^{1+}$, Diana A Tran ${ }^{1,2+}$, Arthur X Li ${ }^{3}$, Charles Warden ${ }^{1}$, Angela Y Bai ${ }^{1,4}$, Purnima Singh ${ }^{1}$, Xiwei Wu', Gerd P Pfeifer ${ }^{5,6}$ and Piroska E Szabó ${ }^{1,6^{*}}$

\begin{abstract}
Background: Exposure to environmental endocrine-disrupting chemicals during pregnancy reportedly causes transgenerationally inherited reproductive defects. We hypothesized that to affect the grandchild, endocrine-disrupting chemicals must alter the epigenome of the germ cells of the in utero-exposed G1 male fetus. Additionally, to affect the great-grandchild, the aberration must persist in the germ cells of the unexposed G2 grandchild.

Results: Here, we treat gestating female mice with vinclozolin, bisphenol A, or di-(2-ethylhexyl)phthalate during the time when global de novo DNA methylation and imprint establishment occurs in the germ cells of the G1 male fetus. We map genome-wide features in purified G1 and G2 prospermatogonia, in order to detect immediate and persistent epigenetic aberrations, respectively. We detect changes in transcription and methylation in the G1 germline immediately after endocrine-disrupting chemicals exposure, but changes do not persist into the G2 germline. Additional analysis of genomic imprints shows no persistent aberrations in DNA methylation at the differentially methylated regions of imprinted genes between the G1 and G2 prospermatogonia, or in the allele-specific transcription of imprinted genes between the $G 2$ and $G 3$ soma.

Conclusions: Our results suggest that endocrine-disrupting chemicals exert direct epigenetic effects in exposed fetal germ cells, which are corrected by reprogramming events in the next generation. Avoiding transgenerational inheritance of environmentally-caused epigenetic aberrations may have played an evolutionary role in the development of dual waves of global epigenome reprogramming in mammals.
\end{abstract}

Keywords: endocrine disruptor, germline epigenetic reprogramming, DNA methylation, vinclozolin, bisphenol A, DEHP, transgenerational epigenetic inheritance, fetal germ cell, genomic imprinting

\section{Background}

Humans are broadly exposed to synthetic endocrinedisrupting chemicals (EDs) from the environment [1-5]. EDs closely resemble endogenous hormones in structure and have been reported to cause developmental and reproductive health problems [6-10]. EDs have the ability to affect gene expression and DNA methylation [11]. It has been suggested that one initial exposure to EDs in

\footnotetext{
* Correspondence: piroska.szabo@vai.org

${ }^{\dagger}$ Equal contributors

'Department of Molecular and Cellular Biology, Beckman Research Institute, City of Hope, Duarte, California 91010, USA

${ }^{6}$ Center for Epigenetics, Van Andel Research Institute, Grand Rapids, Michigan 49503, USA

Full list of author information is available at the end of the article
}

utero harms multiple generations in rodents and that the underlying mechanism is epigenetic [6,12-14]. However, the molecular mechanisms mediating such ED exposuredependent transgenerational inheritance in the germline have not been identified.

In utero ED exposure may harm epigenetic remodeling events (Figure 1) in the germline of the embryo or fetus [15]. In the mouse, such events include global erasure of DNA methylation in primordial germ cells (PGCs) in embryos of both sexes [16-18] and de novo establishment of the sperm-type DNA methylation in fetal male germ cells (MGCs) [19]. De novo DNA methylation in the female germline may be less vulnerable to in utero

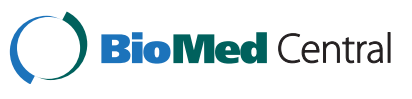

(c) 2015 lqbal et al.; licensee BioMed Central. This is an Open Access article distributed under the terms of the Creative Commons Attribution License (http://creativecommons.org/licenses/by/4.0), which permits unrestricted use, distribution, and reproduction in any medium, provided the original work is properly credited. The Creative Commons Public Domain Dedication waiver (http://creativecommons.org/publicdomain/zero/1.0/) applies to the data made available in this article, unless otherwise stated. 


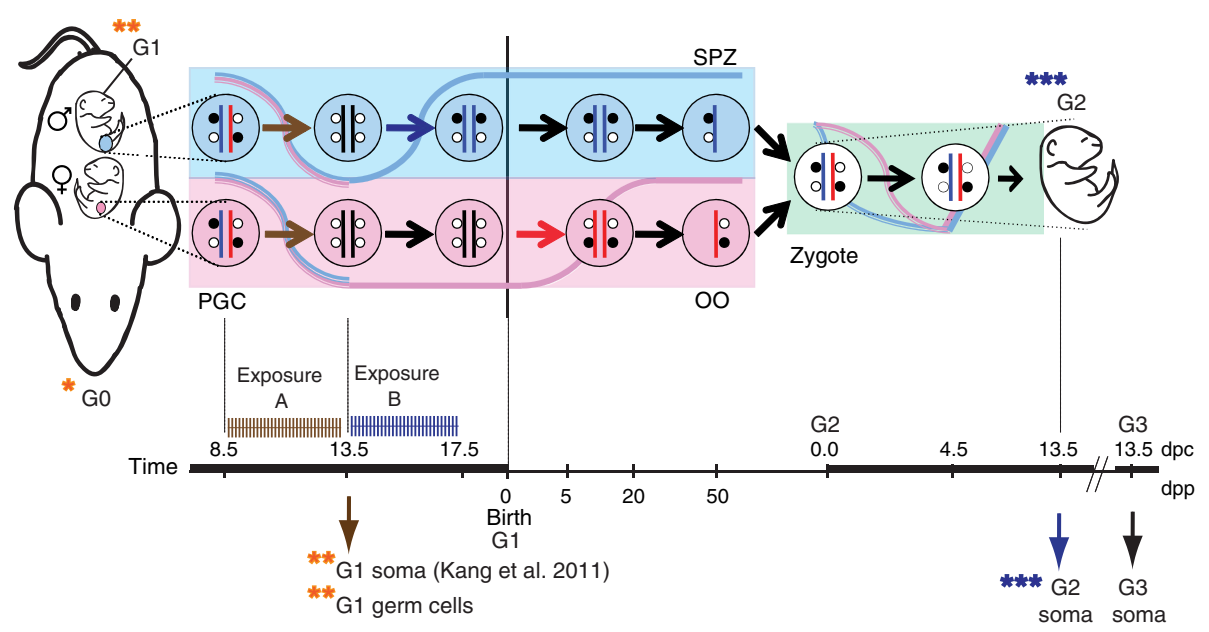

Figure 1 Assessing ED effects on imprint reprogramming in the mouse germline. (Top) Reprogramming in the mouse germline. Exposure of pregnant mice to EDs may directly affect the G0 dam and the G1 soma and G1 germline, and indirectly, the G2 soma that develops from G1 germ cells. DNA methylation patterns are globally remodeled during gametogenesis in both male (above) and female (below) embryos/fetuses in the chromosomes that are paternally (blue) or maternally (red) inherited. The 5-methylcytosine levels (blue and pink curves) are globally reduced in the primordial germ cells (PGC) of both sexes; DNA methylation of imprinted DMRs is similarly erased (brown horizontal arrow) by midgestation $(13.5 \mathrm{dpc})$. In the male germ cells, global DNA methylation is largely reset and paternal imprints are newly established (blue arrow) in prospermatogonia prior to birth and maintained (black horizontal arrows) into spermatozoa (SPZ). In female germ cells, global DNA methylation and maternal imprints are established (red arrow) after birth during oocyte (OO) growth (from 5 to $20 \mathrm{dpp}$ ). The imprinting marks are maintained (black arrows) through global remodeling at fertilization and embryo development. (Bottom) Daily gavage was given to pregnant dams in the time windows of 'Exposure A' or 'Exposure B' to affect the erasure phase of the germline reprogramming in PGCs or the establishment phase in MGC. The timeline (not to scale) is marked with gestational age (dpc) on top, and also with the age after birth (dpp) at the bottom. In our related publication [32], maintenance of imprinting was analyzed in the exposed G1 soma after 'Exposure A'. In the current study, we focused on the effect of EDs on imprinting via the exposed G1 germ cells. Experimental endpoints are indicated by the arrows pointing down.

exposures, as it takes place after birth in the growing oocytes of the juvenile female [20,21].

An important part of the germline remodeling process is the resetting of genomic imprints (Figure 1). Imprinted genes control important developmental processes, including pre- and postnatal growth, metabolism, and behavior. Failure to reprogram imprinted genes in the germ line due to a compromised in utero environment is of special concern [22-24]. Parental allele-specific monoallelic transcription of imprinted genes in the soma mainly depends on the resetting - erasure and re-establishment of their differentially methylated regions (DMRs) in the male and female germlines [25]. DNA methylation marks at DMRs are erased in PGCs in both sexes by 13.5 days post coitum (dpc) (Figure 1) [26,27]. Erasure of imprints results in a shift from monoallelic to biallelic expression of imprinted genes [28,29]. Re-establishment of the malespecific DNA methylation marks occurs at paternally methylated (PAT) DMRs in prospermatogonia and this methylation is thereafter maintained to the spermatozoa stage [30]. Whereas PAT DMRs appear to follow the default global pattern of de novo DNA methylation in prospermatogonia, MAT DMRs in the same cells are protected from de novo DNA methylation at sites of H3K4 methylation [19]. Sperm- or oocyte-specific imprinting marks continue to be maintained following fertilization during the global wave of epigenetic remodeling that takes place in the zygote and early embryo [31] and are further maintained in the soma in the paternally and maternally inherited chromosomes.

Several studies have reported that the process of genomic imprinting is perturbed by endocrine disruptors. The maintenance of imprinting in the in utero exposed embryo is largely resistant but not completely immune to the effects of EDs, as can be seen from the minor aberrations and increased noise in allele-specific transcription of imprinted genes [32]. PGCs at $12.5 \mathrm{dpc}$ exhibit an accelerated imprint erasure rate at the Igf2r, Peg3, and H19 DMRs after in utero BPA exposure [33]. In utero exposure to VZ led to decreased DNA methylation of PAT DMRs in mouse spermatozoa $[6,34,35]$ in the exposed and further generations, suggesting that the establishment and erasure steps were both disturbed. The establishment step of maternal imprints was affected in mouse oocytes: BPA administration to juvenile females caused a reduction in CpG methylation at the Igf2r- and Peg3 MAT DMRs [36]. The maintenance step of genomic imprinting was perturbed in the offspring after BPA exposure of mothers shortly before and during pregnancy [37]. Human severe azoospermia is associated with DNA methylation imprint defects at the H19-IGF2 DMR [38,39], but it is not known whether EDs have a role in these aberrations. 
We hypothesized that any epigenetic aberration causing transgenerationally inherited phenotype in G2 and G3 individuals after in utero exposure of the male G1 germline must meet the following criteria: (1) it is present in the fetal G1 germ cell immediately after exposure; (2) it persists into the G1 gamete; and (3) it persists into the unexposed G2 fetal germ cell of the next generation. In this study, we sought to detect such immediate and persistent aberrations. We analyzed in utero-exposed G1 prospermatogonia and unexposed G2 prospermatogonia to detect immediate and persistent effects of EDs on global transcription. We searched for immediate and persistent changes in DNA methylation in G1 and G2 prospermatogonia and also in G1 and G2 adult spermatozoa. To further analyze the effects of EDs on the imprinting process we also followed the potential germline epigenetic aberration into the G2 and G3 soma. Our data collectively show that the male germline suffers from immediate epigenetic effects after in utero BPA, DEHP, and VZ exposure but recovers in the subsequent generation.

\section{Results}

The aim of this study was to systematically and rigorously evaluate the effects of EDs on global epigenetic reprogramming and imprint resetting in the male germline after in utero exposure. We chose three EDs: vinclozolin(VZ) at $100 \mathrm{mg} / \mathrm{kg} /$ day; di-(2-ethylhexyl) phthalate (DEHP) at $750 \mathrm{mg} / \mathrm{kg} /$ day; and bisphenol A (BPA) at $0.2 \mathrm{mg} / \mathrm{kg} /$ day. These in utero doses have been reported to cause morphological or physiological defects, including reproductive harm in the G1 fetuses (see Methods section).

\section{Testing the effect of EDs on imprint erasure}

We treated pregnant dams daily from $8.5 \mathrm{dpc}$ to 12.5 $\mathrm{dpc}$ (exposure A in Figure 1) with one of the EDs or with corn oil as control. For this experiment we used JF1 females and OG2 males (Figure 2A). The OG2 transgenic line carries an Oct4 promoter-GFP transgene that allowed us to purify the GFP-positive male and female germ cells (MGCs and FGCs) and GFP-negative somatic cells (MSCs and FSCs) from the dissected embryonic gonads by FACS sorting (Figure 2B). The JF1 inbred mouse strain is genetically distinct from the OG2 line, providing single nucleotide polymorphisms. This allowed measurement of the allele-specific transcription of known imprinted genes in JF1 $\times$ OG2 cells using multiplex RNA-single nucleotide primer extension (SNuPE) assays uisng Sequenom allelotyping [32].

We observed correct parental allele-specific transcription in control-treated FSCs and MSCs at $13.5 \mathrm{dpc}$ for ubiquitously imprinted transcripts; for example, Kcnq1ot1, Peg3, Plagl1, and Dlk1 were expressed from the paternal OG2 allele, and Igf2r, H19, and Meg3 were expressed from the maternal JF1 allele (Figure 2C and Additional file 1).
These expression patterns represent the normal correct ubiquitous parental allele-specific transcription. Some other transcripts (Kcnq1, Phlda2, and Atp10a, for example, which have tissue-specific or developmental stage-specific imprinting) displayed biallelic transcription in FSCs and MSCs. The parental allele-specific transcription in PGCs at $9.5 \mathrm{dpc}$ switched to biallelic transcription in control FGCs and MGCs at $13.5 \mathrm{dpc}$, consistent with the erasure of genomic imprints.

Looking at the erasure of allele-specific transcription in ED-treated FGCs and MGCs, we found few aberrations that were greater than $5 \%$ and were statistically significant (Student's $t$-test, $P<0.05)$ (Table 1 ). Meg3 was affected by BPA in FGCs and by VZ in MGCs; H19 was affected by DEHP in FGCs; and Zim1 was affected by DEHP in FGCs. Considering the number of tests $(28$ SNPs tested), we could expect one or two positive results for each ED in each cell type simply by chance $(P$ $<0.05)$; the number we found was close to that expectation. Nesp and Asb4 appeared to have a change, but these genes did not exhibit correct parental allelespecific transcription in PGCs (Figure 2C). Therefore, these cannot be considered as erasure defects. Using multiple testing, we found that none of the erasure defects were significant for any of the EDs using the Bonferroni corrected $P$ value $(P<0.05 / 28=0.0018)$.

\section{Effect of EDs on allele-specific DMR methylation in embryos derived from exposed prospermatogonia}

In utero ED exposure can perturb the imprinting process in fetal germ cells at the time when genomic imprints are established at PAT DMRs and when MAT DMRs are protected from de novo DNA methylation. Such perturbation in the normal imprint establishment process in male germ cells would result in reduced de novo methylation at PAT DMRs in the prospermatogonia of G1 fetuses and reduced methylation in the paternally inherited allele in the soma of the G2 generation. Changes at MAT DMRs would mean lack of protection from DNA methylation in G1 prospermatogonia and consequently their increased methylation in the paternally inherited chromosome in the G2 generation. We anticipated that inherited epigenetic changes would result in aberrant DNA methylation of the same DMR in more than one organ.

To test whether ED exposure perturbs the imprinting process in the prospermatogonia, we first tested the allelespecific DNA methylation pattern at DMRs in the soma of the derived offspring. G1 129S1 male offspring were exposed in utero at the time of paternal imprint establishment (exposure B in Figure 1) on five consecutive days, 12.5 to $16.5 \mathrm{dpc}$, by oral gavage to pregnant G0 dams with one of the EDs or vehicle control (Figure 3A). When 
A

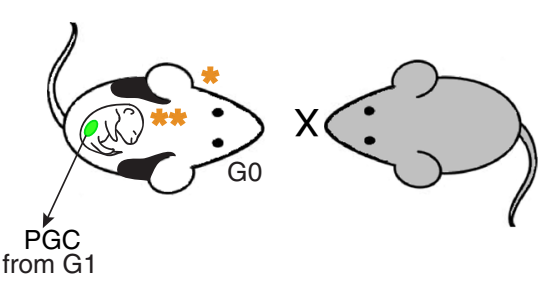

B

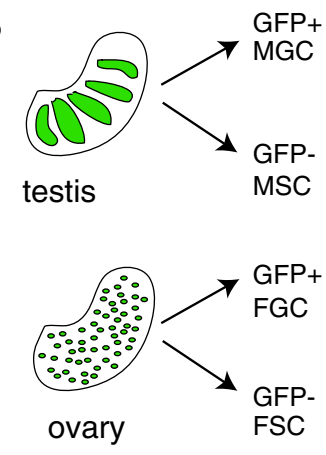

C

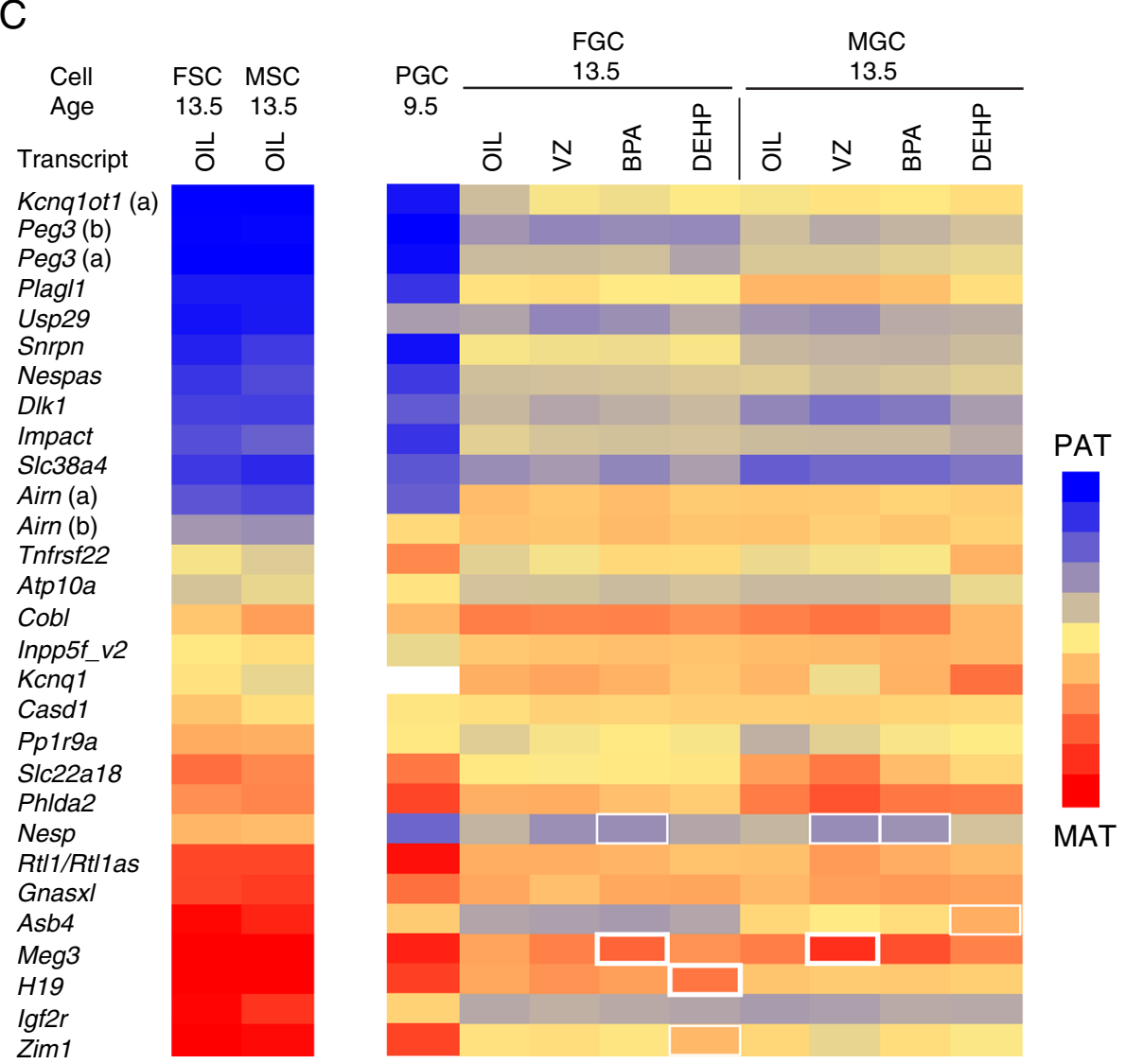

Figure 2 Testing the effect of endocrine-disrupting chemicals (EDs) on imprint erasure. (A) Experimental design. JF1 females (tail up) were mated with OG2 males (tail down). Pregnant dams (G0) were treated with EDs or oil control from $8.5 \mathrm{dpc}$ to $12.5 \mathrm{dpc}$. Imprint erasure occurs during this time in PGCs of the exposed G1 embryos. One orange star = exposed G0 generation; two orange stars = exposed G1 embryo. (B) GFP positive male and female germ cells and somatic cells were collected from the gonads by FACS sorting. (C) Results of RNA Sequenom allelotyping experiments of imprinted transcripts are displayed for cells treated with an ED or control vehicle ('oil'); letters in parentheses indicate separate SNPs. Average $(n=3)$ allele-specific transcription is shown in color, 100\% paternal (blue) to 100\% maternal (red), with 50\% biallelic in yellow. Many imprinted genes have parental allele-specific transcription in PGCs at $9.5 \mathrm{dpc}$ but biallelic transcription in FGCs and MGCs (but not in FSCs and MSCs) at $13.5 \mathrm{dpc}$. Statistically significant $(P<0.05)$ aberrations in erased allele-specific transcription, greater than $5 \%$ or $10 \%$, are marked by thin or bold white rectangles, respectively.

129S1 exposed G1 males reached adulthood, they were mated with unexposed JF1 females to generate G2 offspring derived from the exposed prospermatogonia. The JF1 $\times 129$ G2 fetuses were dissected at $13.5 \mathrm{dpc}$ to collect head, heart, liver, lung, placenta, yolk sac, and embryo carcass. We isolated DNA and collected the CpG-methylated fraction using MIRA [40]. We quantified the percentage of parental alleles in the total methylated fraction at 14 DMRs (18 SNPs) using multiplex SNuPE assays [41]. The average percentage 
Table 1 Significant changes between control and ED-treated samples after disturbing G1 germ line imprint erasure - RNA

\begin{tabular}{|c|c|c|c|c|c|c|c|c|}
\hline \multirow[b]{2}{*}{ Transcript } & \multirow[b]{2}{*}{ Cell type } & \multirow[b]{2}{*}{ ED } & \multirow[b]{2}{*}{ Difference (\%) } & \multirow[b]{2}{*}{ SD (\%) } & \multirow[b]{2}{*}{ Student's $t$-test $P$ value } & \multirow[b]{2}{*}{ Imprinted base line (cutoff $80 \%$ ) } & \multicolumn{2}{|c|}{$\%$ MAT allele Avg. $(n=3)$} \\
\hline & & & & & & & Oil & Treatment \\
\hline Nesp & MGC & VZ & -9.1 & 3.440 & 0.004 & NO (erased) & 39 & 30 \\
\hline Nesp & FGC & BPA & -8.2 & 4.356 & 0.042 & NO (erased) & 38 & 30 \\
\hline Nesp & MGC & BPA & -7.9 & 0.956 & 0.001 & NO (erased) & 39 & 31 \\
\hline Asb4 & MGC & DEHP & 8.7 & 3.769 & 0.049 & NO (erased) & 54 & 63 \\
\hline Ziml & FGC & DEHP & 9.0 & 5.406 & 0.030 & NO (erased) & 52 & 61 \\
\hline H19 & FGC & DEHP & 10.6 & 7.507 & 0.024 & NO (erased) & 65 & 75 \\
\hline Meg3 & FGC & BPA & 13.7 & 4.713 & 0.016 & NO (erased) & 65 & 79 \\
\hline Meg3 & MGC & VZ & 16.8 & 4.438 & 0.002 & NO (erased) & 73 & 90 \\
\hline
\end{tabular}

Allele-specific transcription was compared between ED- and vehicle-treated samples for each transcript in 13.5 dpc FGC and MGC using SNuPE assays. Changes in the average $(n=3)$ allele-specific transcription that were greater than $5 \%$ and were statistically significant $(P<0.05)$ were tabulated and ordered according to the difference in maternal allele-specific expression. Baseline allele specificity of transcription in the maternal (MAT) or paternal (PAT) allele was not observed (NO) in the vehicle-treated sample (cutoff $80 \%$ ), indicating erased imprinting in PGCs. Of those with baseline erasure, the expression or DNA methylation became more biased toward one parental allele in a few instances, indicating a lack of proper erasure of imprinted expression (see the last column).

of paternal or maternal allele methylation is displayed in Figure 3C and Additional file 1. From 378 different experimental conditions (7 organs, 3 EDs, 18 SNPs), we found only four statistically significant differences $(P<0.05)$ that were greater than 5\% (Table 2), and no difference was higher than $10 \%$. The CpG methylation was less paternally biased in G2 embryos at $13.5 \mathrm{dpc}$ at the IG-DMR in the lung; the Peg1-Mest, Nespas, and Slc38a4 DMRs were less maternally biased in the head, embryo carcass, and lung, respectively, after VZ treatment of the prospematogonia of G1 fetuses. Applying multiple testing, none of these changes were significant using the Bonferroni corrected $P$ value $(P<0.0027)$. Additional SNPs in two of these DMRs (IG-DMR and Nespas) did not substantiate the epigenetic aberration. We did not detect any change in the G2 soma after BPA or DEHP treatment of $\mathrm{G} 1$ prospermatogonia.

We also carried out the control experiment to test whether in utero EDs perturb the imprinting process in female fetal germ cells, which would result in increased de novo methylation at PAT DMRs in primary oocytes of G1 fetuses. That methylation could be carried into the soma of G2 generation in the maternally inherited allele of PAT DMRs. Perturbation at MAT DMRs would cause earlier DNA methylation in oocytes but would not be expected to change the final DNA methylation levels of the oocyte nor to increase DNA methylation in the maternally inherited chromosome in the G2 generation. We exposed the 129S1 G1 female offspring to an ED or vehicle control (Figure 3B) in utero. We crossed G1 adult females with unexposed JF1 males to generate G2 offspring, deriving from the exposed primary oocytes. We dissected the $\mathrm{G} 2$ fetuses at $13.5 \mathrm{dpc}$ and collected seven organs/body parts for DNA isolation. We measured the parental allelespecific DNA methylation at 14 DMRs using
MIRA-SNuPE assays (Figure 3D and Additional file 1 ). We found five significant changes in the maternal allele-specific methylation $(>5 \%, P<0.05)$ at PAT DMRs (Table 2). The IG-DMR in the liver and head was affected by VZ exposure; the Rasgrf1 DMR was affected in the head and heart by DEHP and in the head by VZ exposure of fetal oocytes. One of these, the IG-DMR in G2 liver was significant after Bonferroni correction of $P$ value to $P<0.0027$. However, this aberration was not substantiated by another SNP in the same DMR.

We found 10 cases in which maternal allele-specific methylation decreased at MAT DMRs by at least $5 \%$ $(P<0.05)$, and two remained significant after Bonferroni adjustment $(P<0.0027)$. However, these aberrations in $\mathrm{G} 2$ soma cannot be explained by disturbed DNA methylation in fetal oocytes. Another layer of epigenetic regulation may have been disturbed to prevent the full de novo DNA methylation of MAT DMRs in growing oocytes after the birth of the G1 females. It is quite possible that ED exposure in G0 affects the ability of G1 females to reproduce by altering the uterine environment, and thus it may affect G2 independent of the germline. Since this experimental model is not as 'clean' when addressing germline inheritance as the male inheritance model (where there is no confounding uterine environment effect), these data should be interpreted with caution.

\section{Effect of EDs on allele-specific expression of imprinted genes in embryos derived from exposed prospermatogonia}

We expected that any epigenetic aberration - DNA methylation or other - occurring in G1 prospermatogonia at PAT or MAT DMRs would result in misexpression of imprinted genes in the soma of the G2 generation. To test for this possibility, we followed the experimental design shown in Figure 3A and measured parental allele-specific 

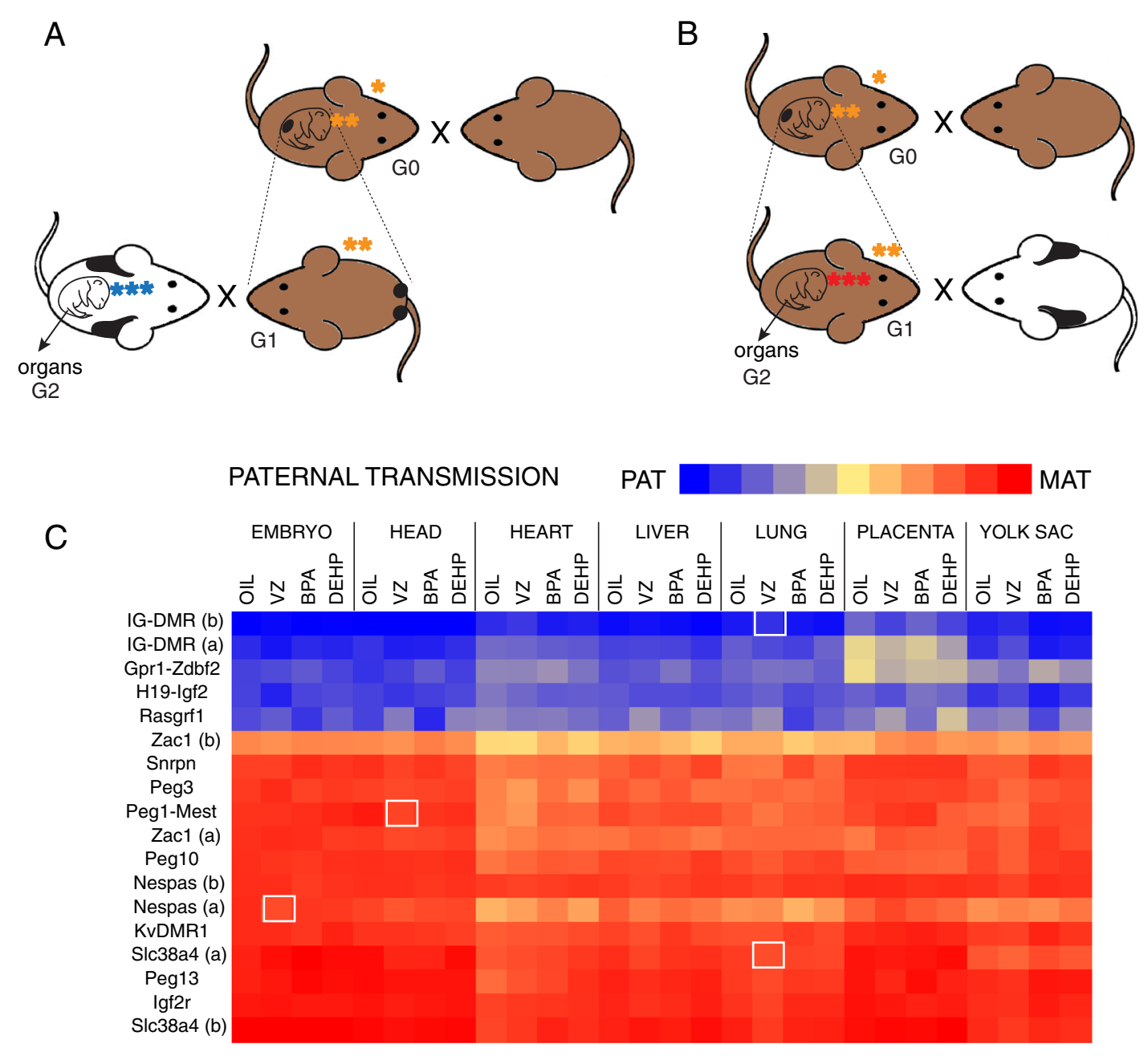

C

D

\section{MATERNAL TRANSMISSION}

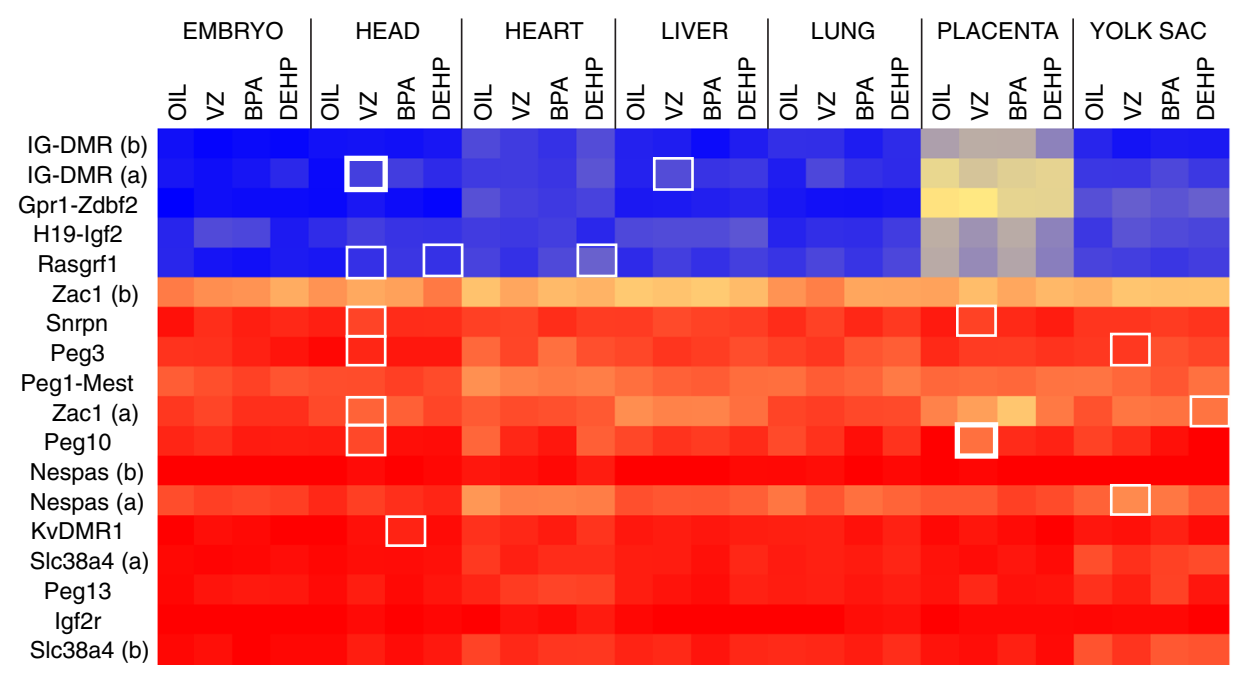

Figure 3 (See legend on next page.) 
(See figure on previous page.)

Figure 3 Testing the effect of EDs on imprint establishment by assaying the allele-specific DNA methylation at DMRs in G2 embryos.

(A) In utero exposed male 129S1 G1 fetuses were grown to adulthood and mated with unexposed JF1 females; the resulting G2 fetuses

(three blue stars) derived from exposed prospermatogonia. (B) In utero exposed G1 females were mated with unexposed JF1 males to generate G2 offspring (three red stars) which derived from exposed primary oocytes. G2 fetuses were dissected at 13.5 dpc to collect organs.

(C, D) MIRA-SNuPE results of $13.5 \mathrm{dpc}$ G2 embryo DNA samples after potential paternal and maternal transmission of aberrant DNA methylation at DMRs. The heatmap shows the percentage of average parental allele-specific methylation in organs; the color scale is as in Figure $2 \mathrm{C}$. Letters in parentheses indicate different SNPs. Statistically significant differences relative to control (oil) greater than $5 \%$ and $10 \%$ are indicated by thin or bold rectangles, respectively (Student's t-test, $P<0.05$ ).

transcription in G2 embryo organs by RNA-SNuPE using multiplex Sequenom allelotyping assays at 56 SNPs. The results are provided in Additional file 1 and are displayed in two heatmaps (Figure 4). Analyzing three G2 individuals from each treatment, seven organs, and 56 SNPs, we generated 4,704 data points and compared the average values, finding five differences greater than $5 \%$ relative to control (Student's $t$-test, $P<0.05$ ). None of these differences remained significant after multiple testing using Bonferroni correction of $P$ values $(P<0.0009)$. To test for possible inter-litter variations, we repeated this experiment with a larger number of G2 fetuses, selected organs, and selected SNPs, generating 1,350 data points (Additional files 1 and $2 \mathrm{~A}$ ); there were no differences greater than $5 \%$ relative to control $(P<0.05)$. We conclude that allele-specific transcription was undisturbed in the G2 embryos derived from ED-exposed prospermatogonia.

We found no causative relationship between aberrations in allele-specific DNA methylation at DMRs and allelespecific transcription. For example, the IG-DMR was affected by VZ in lung (Figure 3C), but transcription of Meg3, Rtl1/Rtl1as, Rian, and Rian extension, all regulated by IG-DMR, were not altered there (Figure 4). Similarly, the Nespas DMR was altered by VZ in the embryo carcass, but the allele-specific transcription of Nesp, Nespas, and Gnasxl showed no change.

\section{Testing for transgenerational inheritance of aberrant imprinting}

There were a few statistically significant perturbations in the transcription of imprinted genes in G2 heart and lung greater than $5 \%$ (Figure 4). We asked whether these could be passed from G2 individuals into the unexposed G3 generation. Our experimental design is shown in Figure 5A. We treated G1 male offspring in utero daily from $12.5 \mathrm{dpc}$ to $16.5 \mathrm{dpc}$, when paternal imprint is occurring in prospermatogonia, with an ED or with vehicle control ('oil'). We crossed adult G1 129S1 males with unexposed 129S1 females and allowed G2 males to reach adulthood. Then we crossed them with unexposed JF1 females to generate G3 offspring. Whereas G2 fetuses developed from ED-exposed germ cells, G3 fetuses developed from unexposed gametes.
We dissected the heart and lung of JF1 $\times 129$ G2 fetuses at $13.5 \mathrm{dpc}$ and we quantified parental-specific transcription in the total RNA using multiplex SNuPE assays (Figure 5B and Additional file 1).

Changes that occurred in the G2 generation did not carry into G3. We found no statistically significant change $(P<0.05)$ greater than $5 \%$ in the paternal allele-specific transcription of Usp29 in the heart (BPA) or Rasgrf1 in the lung (VZ). We found three statistically significant changes (in Snrpn, Nespas, and Rtl1/Rtl1as) in G3 lung after VZ exposure of G1 prospermatogonia (Table 2), but these must be independent of the initial VZ exposure, because we did not find any misexpression of their transcripts in the G2 embryo. To test for interlitter variation, we repeated this experiment using a larger number of G3 fetus livers and selected SNPs (Additional files 1 and $2 \mathrm{~B}$ ). In this dataset, we did not detect any difference greater than $5 \%$ as relative to oil control $(P<0.05)$. Thus, our results do not support the hypothesis that ED-triggered epigenetic aberrations in imprint establishment of prospermatogonia are transgenerationally inherited.

\section{Effect of EDs on allele-specific expression of imprinted} genes in embryos derived from exposed primary oocytes We used the experimental design shown in Figure 3B to expose G1 129S1 female offspring to an ED or vehicle control in utero. We crossed G1 adult females with unexposed JF1 males to generate G2 offspring that were from exposed primary oocytes. We dissected JF1 $\times 129$ G2 fetuses at $13.5 \mathrm{dpc}$, collected seven organs/body parts for RNA isolation, and measured the parental allele-specific DNA methylation at 33 SNPs using RNA-SNuPE assays (Additional files 1 and 3). We found four significant decreases in maternal allele-specific transcription of more than 5\% (Table 2): Slc22a3 and Gnasxl in the yolk sac after DEHP exposure, Nesp in the placenta (DEHP), Ascl2 in the liver (VZ). None of these remained statistically significant after multiple testing using the Bonferroni corrected $P$ value $(P<0.0015)$. We found no causative relationship between aberrant allele-specific DNA methylation at DMRs and aberrant allele-specific transcription (Figure 3D and Additional file 3). 
Table 2 Significant changes between control and ED-treated samples after disturbing G1R DMR establishment

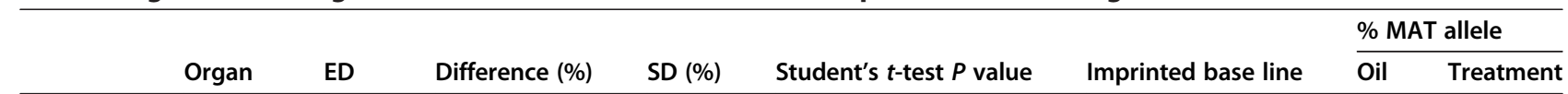

G2 soma trough paternal germ line - DMR DNA methylation (Figure 3C)

$\begin{array}{llllll}\text { Peg1-Mest } & \text { Head } & \text { VZ } & -9.1 & 2.523 & 0.038 \\ \text { Nespas } & \text { Embryo } & \text { VZ } & -6.9 & 3.150 & 0.022 \\ \text { SIC38a4 (b) } & \text { Lung } & \text { VZ } & -5.9 & 1.747 & 0.006 \\ \text { IG-DMR } & \text { Lung } & \text { VZ } & 5.1 & 1.513 & 0.010\end{array}$

$\begin{array}{lll}\text { YES (MAT) } & 95 & 86 \\ \text { YES (MAT) } & 91 & 84 \\ \text { YES (MAT) } & 94 & 88 \\ \text { YES (PAT) } & 5 & 10\end{array}$

G2 soma through maternal germ line - DMR DNA methylation (Figure 3D)

$\begin{array}{llllll}\text { Peg10 } & \text { Placenta } & \text { VZ } & -23.4 & 3.247 & 0.000 \\ \text { Peg10 } & \text { Head } & \text { VZ } & -9.5 & 2.146 & 0.035 \\ \text { Nespas (a) } & \text { Yolk sac } & \text { VZ } & -8.5 & 4.049 & 0.023 \\ \text { Snrpn } & \text { Placenta } & \text { VZ } & -8.4 & 4.675 & 0.049 \\ \text { Snrpn } & \text { Head } & \text { VZ } & -7.7 & 2.521 & 0.020 \\ \text { Zacl (a) } & \text { Yolk sac } & \text { DEHP } & -7.3 & 2.129 & 0.037 \\ \text { KVDMR1 } & \text { Head } & \text { BPA } & -7.2 & 1.497 & 0.001 \\ \text { Peg3 } & \text { Head } & \text { VZ } & -6.4 & 2.483 & 0.034 \\ \text { Zac1 (a) } & \text { Placenta } & \text { VZ } & -5.9 & 3.248 & 0.036 \\ \text { Zacl (b) } & \text { Placenta } & \text { VZ } & -5.7 & 2.239 & 0.018 \\ \text { Peg3 } & \text { Yolk sac } & \text { VZ } & -5.6 & 2.541 & 0.022 \\ \text { Zac1 (a) } & \text { Head } & \text { VZ } & -5.5 & 1.528 & 0.036 \\ \text { Rasgrf1 } & \text { Head } & \text { VZ } & 5.4 & 1.343 & 0.003 \\ \text { Rasgrf1 } & \text { Head } & \text { DEHP } & 5.5 & 2.038 & 0.011 \\ \text { Rasgrf1 } & \text { Heart } & \text { DEHP } & 6.4 & 1.413 & 0.003 \\ \text { IG-DMR (a) } & \text { Liver } & \text { VZ } & 9.0 & 1.087 & 0.002 \\ \text { IG-DMR (a) } & \text { Head } & \text { VZ } & 11.2 & 3.210 & 0.048\end{array}$

$\begin{array}{lll}\text { YES (MAT) } & 100 & 77 \\ \text { YES (MAT) } & 95 & 85 \\ \text { YES (MAT) } & 80 & 71 \\ \text { YES (MAT) } & 95 & 86 \\ \text { YES (MAT) } & 94 & 86 \\ \text { YES (MAT) } & 83 & 76 \\ \text { YES (MAT) } & 100 & 93 \\ \text { YES (MAT) } & 99 & 92 \\ \text { NO } & 73 & 67 \\ \text { NO } & 66 & 61 \\ \text { YES (MAT) } & 88 & 83 \\ \text { YES (MAT) } & 85 & 79 \\ \text { YES (PAT) } & 6 & 12 \\ \text { YES (PAT) } & 6 & 12 \\ \text { YES (PAT) } & 15 & 22 \\ \text { YES (PAT) } & 9 & 18 \\ \text { YES (PAT) } & 4 & 15\end{array}$

G2 soma through paternal germ line - RNA transcripts (Figure 4)

$\begin{array}{llllll}\text { Nesp } & \text { Lung } & \text { DEHP } & -14.3 & 2.166 & 0.001 \\ \text { Nesp } & \text { Heart } & \text { DEHP } & -11.9 & 3.157 & 0.042 \\ \text { Slc22a3 } & \text { Head } & \text { VZ } & -7.8 & 2.097 & 0.027 \\ \text { Asc12 } & \text { Liver } & \text { VZ } & -7.1 & 3.323 & 0.043 \\ \text { Cdkn1c } & \text { Embryo } & \text { BPA } & -6.7 & 2.865 & 0.036 \\ \text { Slc22a3 } & \text { Liver } & \text { VZ } & -6.0 & 2.261 & 0.011 \\ \text { Slc22a3 } & \text { Liver } & \text { BPA } & -6.0 & 2.925 & 0.025 \\ \text { Cdkn1C } & \text { Embryo } & \text { VZ } & -5.8 & 1.367 & 0.021 \\ \text { Cdkn1c } & \text { Embryo } & \text { DEHP } & -5.8 & 0.535 & 0.014 \\ \text { Slc22a3 } & \text { Lung } & \text { BPA } & -5.7 & 2.710 & 0.031 \\ \text { Usp29 } & \text { Lung } & \text { BPA } & 5.7 & 1.424 & 0.040 \\ \text { Gnasxl } & \text { Heart } & \text { VZ } & 6.3 & 1.940 & 0.027 \\ \text { Rasgrf1 } & \text { Heart } & \text { VZ } & 9.0 & 0.722 & 0.006 \\ \text { Gnasxl } & \text { Lung } & \text { VZ } & 15.3 & 3.644 & 0.002 \\ \text { G2 soma th } & & & \end{array}$

G2 soma through maternal germ line - RNA transcripts (Additional file 3)

$\begin{array}{llllll}\text { Nesp } & \text { Placenta } & \text { DEHP } & -12.7 & 4.922 & 0.034 \\ \text { Slc22a3 } & \text { Yolk sac } & \text { DEHP } & -11.0 & 0.620 & 0.030 \\ \text { Asc12 } & \text { Liver } & \text { VZ } & -9.4 & 1.324 & 0.034 \\ \text { Gnasxl } & \text { Yolk sac } & \text { DEHP } & -8.6 & 3.204 & 0.035\end{array}$

$\begin{array}{lll}\text { NO } & 45 & 31 \\ \text { NO } & 45 & 33 \\ \text { NO } & 53 & 46 \\ \text { NO } & 77 & 70 \\ \text { YES (MAT) } & 99 & 92 \\ \text { NO } & 57 & 51 \\ \text { NO } & 57 & 51 \\ \text { YES (MAT) } & 99 & 93 \\ \text { YES (MAT) } & 99 & 93 \\ \text { NO } & 64 & 58 \\ \text { YES (PAT) } & 3 & 9 \\ \text { NO } & 26 & 32 \\ \text { YES (PAT) } & 18 & 27 \\ \text { NO } & 31 & 46\end{array}$


Table 2 Significant changes between control and ED-treated samples after disturbing G1R DMR establishment (Continued)

\begin{tabular}{|c|c|c|c|c|c|c|c|c|}
\hline \multicolumn{9}{|c|}{ G3 soma through paternal germ line (lung and heart) - RNA transcripts (Figure 5B) } \\
\hline Cobl & Heart & BPA & -28.0 & 2.289 & 0.004 & $\mathrm{NO}$ & 79 & 51 \\
\hline Cobl & Heart & VZ & -27.9 & 0.640 & 0.001 & NO & 79 & 51 \\
\hline Cobl & Lung & VZ & -19.7 & 2.033 & 0.005 & $\mathrm{NO}$ & 70 & 51 \\
\hline Cobl & Lung & BPA & -19.4 & 2.729 & 0.018 & $\mathrm{NO}$ & 70 & 51 \\
\hline Phlda2 & Lung & VZ & -11.8 & 3.974 & 0.032 & NO & 79 & 68 \\
\hline Cobl & Heart & DEHP & -11.1 & 1.206 & 0.003 & NO & 79 & 68 \\
\hline Rtl1/Rt/1as & Lung & BPA & -10.0 & 1.649 & 0.021 & YES (MAT) & 90 & 80 \\
\hline Tnfrsf22 & Heart & VZ & -9.9 & 1.942 & 0.026 & NO & 58 & 48 \\
\hline Rt/1/Rt/1as & Lung & VZ & -8.7 & 4.378 & 0.007 & YES (MAT) & 90 & 81 \\
\hline Tnfrsf22 & Heart & BPA & -8.1 & 1.070 & 0.022 & NO & 58 & 50 \\
\hline Tnfrsf22 & Lung & BPA & -7.6 & 1.305 & 0.023 & $\mathrm{NO}$ & 57 & 49 \\
\hline $\mathrm{Ascl} 2$ & Heart & DEHP & -6.2 & 2.372 & 0.011 & $\mathrm{NO}$ & 54 & 48 \\
\hline Tnfrsf22 & Lung & VZ & -5.5 & 2.043 & 0.025 & $\mathrm{NO}$ & 57 & 51 \\
\hline Atp10a & Heart & VZ & 5.1 & 1.285 & 0.010 & $\mathrm{NO}$ & 48 & 53 \\
\hline Sfmbt2 & Lung & VZ & 6.5 & 0.690 & 0.003 & $\mathrm{NO}$ & 41 & 48 \\
\hline Ziml & Lung & BPA & 6.7 & 1.473 & 0.010 & YES (MAT) & 90 & 97 \\
\hline Snrpn & Lung & VZ & 9.9 & 2.488 & 0.019 & YES (PAT) & 11 & 21 \\
\hline S/c22a3 & Lung & BPA & 10.0 & 7.898 & 0.017 & NO & 50 & 60 \\
\hline Nespas (a) & Lung & VZ & 10.3 & 2.375 & 0.036 & YES (PAT) & 3 & 13 \\
\hline Ascl2 & Lung & BPA & 12.7 & 3.520 & 0.000 & NO & 46 & 59 \\
\hline Asb4 & Heart & VZ & 27.2 & 8.101 & 0.019 & NO & 61 & 88 \\
\hline Asb4 & Heart & BPA & 27.2 & 2.591 & 0.015 & NO & 61 & 88 \\
\hline
\end{tabular}

Note: (a) and (b) denote different SNPs for the given DMR or transcript.

Allele-specific DNA methylation or transcription was compared in $13.5 \mathrm{dpc}$ embryos between ED- and vehicle-treated samples for each of the DMRs or imprinted transcripts, respectively. Changes in the average $(n=3)$ allele-specific features that were greater than $5 \%$ and were statistically significant $(P<0.05)$ were tabulated and ordered according to the change in the maternal allele. Changes greater than $10 \%$ were rare. Baseline allele specificity of transcription or DNA methylation was observed (YES) or was not observed (NO) in the maternal (MAT) or paternal (PAT) allele in the vehicle-treated sample (cutoff $80 \%$ ). Of those with baseline allele specificity, the expression or DNA methylation became less biased (relaxed) in a few instances (see the last column).

DNA methylation establishment is undisturbed by EDs in prospermatogonia at DMRs

To test ED effects directly, we used the methylated CpG island recovery assay (MIRA) chip. We showed previously that MIRA-chip is sensitive to detect allele-specific DNA methylation at DMRs in mouse embryo fibroblasts (MEFs) [42] and to reveal dynamic DNA methylation changes at DMRs during fetal male germ cell development [19].

To detect immediate changes in DNA methylation in male germ cells at the time of exposure and when the changes are passed to the next generation, we mapped the methylation patterns from the in utero-exposed prospermatogonia and the emerging adult spermatozoa (Figure 6). After crossing a FVB dam and an OG2 father, we treated G1 male fetuses in utero from $12.5 \mathrm{dpc}$ to $16.5 \mathrm{dpc}$ to daily exposure to an ED or to vehicle control at the time of paternal imprint establishment in the prospermatogonia (Additional file 4A). We collected the exposed prospermatogonia at $17.5 \mathrm{dpc}$ for DNA methylation analysis by FACS sorting. We allowed some G1 males to reach adulthood and collected their sperm (Additional file 4A). We isolated genomic DNA from the prospermatogonia and sperm and then we enriched for the methylated fraction using MIRA. We labeled the methylated fraction and hybridized it to custom Nimblegen microarrays that encompassed all known imprinted domains, as we did earlier [19].

The methylation levels of PAT- and MAT-imprinted DMRs in prospermatogonia after ED treatment are shown in Figure 7A. In the controls ('oil'), DNA methylation reprogramming occurred normally: G1-specific DNA methylation was erased and the reprogrammed (G1R)type DNA methylation establishment was largely complete (Additional file 5) [19]. DNA methylation was similarly normal at imprinted DMRs in G1R sperm that developed from VZ-exposed G1R prospermatogonia (Figure 7B) and in ED-exposed G1R prospermatogonia (Figure 7A 


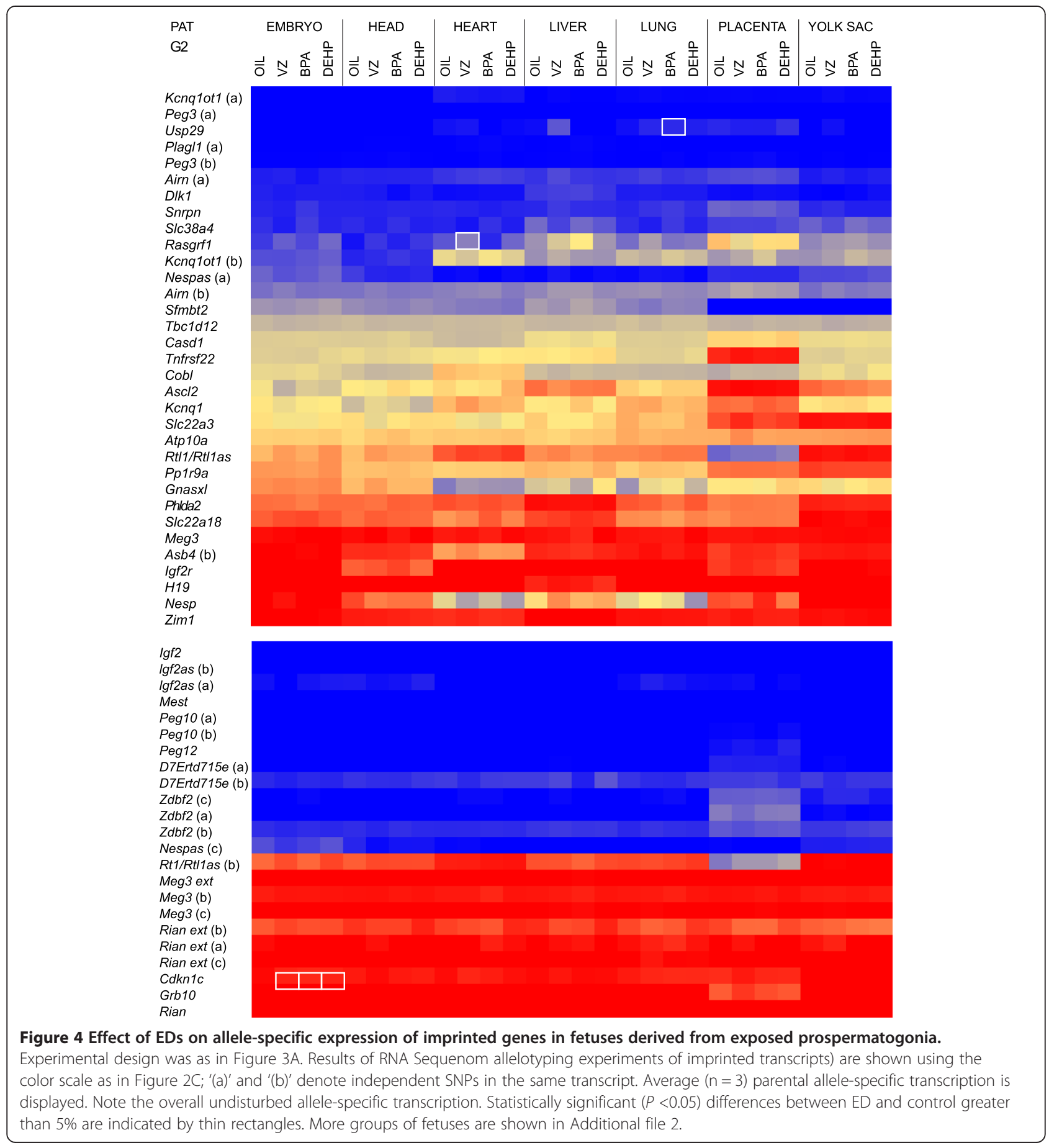

and Additional file 6). Strong peaks were observed at PAT DMRs (Additional file 5A) and valleys were found at MAT DMRs (Additional file 5B); none of these DMRs showed any change after ED exposure.

We also tested the G2R male germ cells of the next unexposed generation derived from exposed G1R propermatogonia (Additional file $4 \mathrm{C}$ and $\mathrm{D}$ ). We exposed G1 male fetuses in utero daily from $12.5 \mathrm{dpc}$ to $16.5 \mathrm{dpc}$ to an ED or vehicle control. We allowed some
G1 (FVBXFVB) males to reach adulthood and crossed them with OG2 females to obtain G2 fetuses (Additional file 4C). These fetuses derived from exposed G1R prospermatogonia, but their prospermatogonia that carried the G2R-type DNA methylation pattern had never been exposed to EDs. We collected the G2R-type prospermatogonia for DNA methylation analysis, and we allowed some VZ-exposed G2 (FVBXOG2) males to reach adulthood and collected their G2R-type 

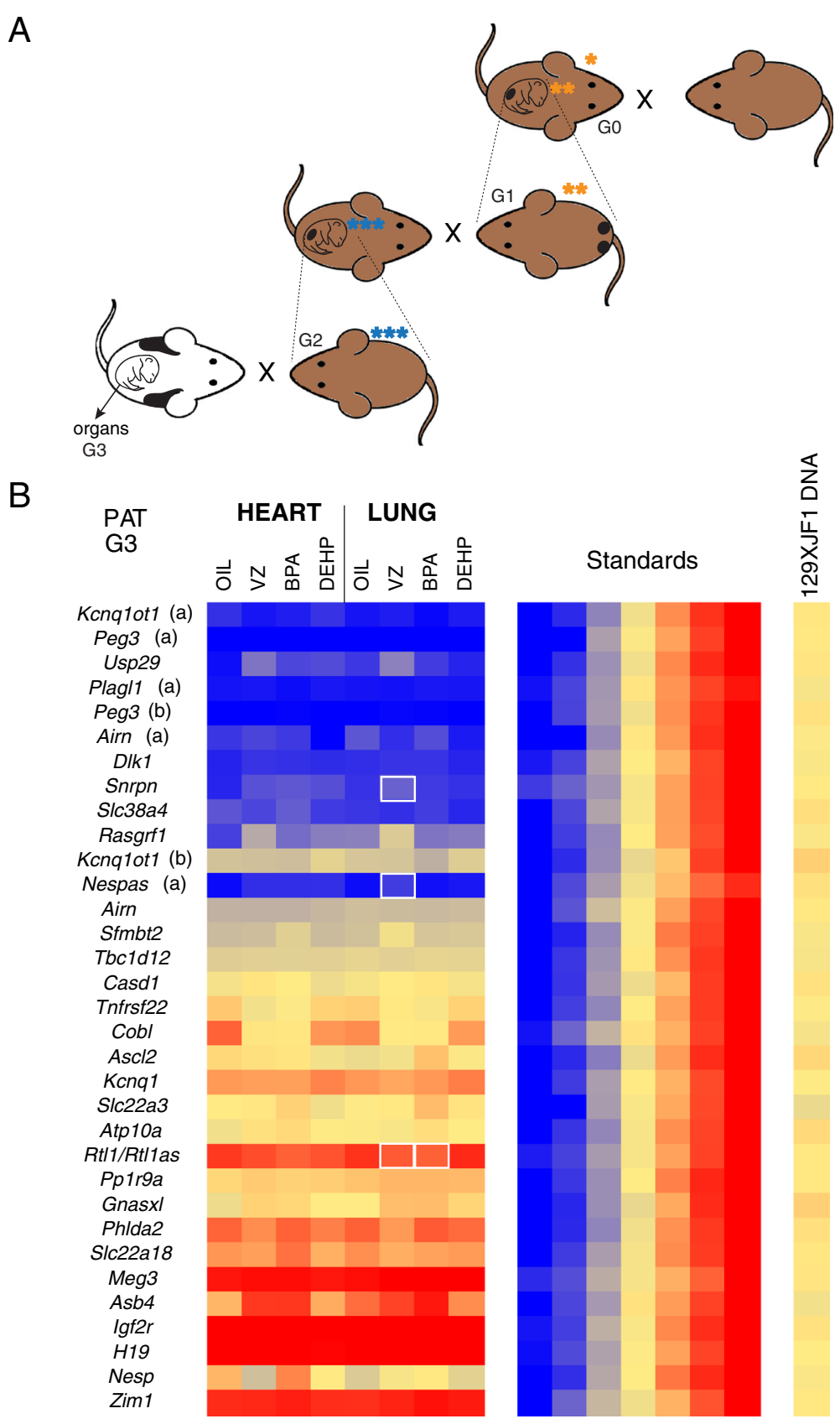

Figure 5 Testing for transgenerational epigenetic inheritance of the aberrant imprinted expression. (A) Breeding design to test whether ED-perturbed parental allele-specific transcription is transgenerationally inherited through the paternal germline to an unexposed generation. G1 male fetuses were exposed in utero to EDs or vehicle control ('oil') daily from $12.5 \mathrm{dpc}$ to $16.5 \mathrm{dpc}$. After reaching adulthood, $129 \mathrm{~S} 1 \mathrm{G} 1 \mathrm{males}$ were mated with $129 \mathrm{~S} 1$ unexposed females to generate G2 offspring (3 blue stars), which derived from exposed prospermatogonia. At adulthood, G2 males were mated with unexposed JF1 females to generate G3 offspring, which were never directly exposed to EDs. JF1 × 129 G3 fetuses were dissected at $13.5 \mathrm{dpc}$ to collect organs for RNA isolation. Parental-specific transcription was quantified in the total RNA using multiplex SNuPE assays. (B) Results of Sequenom allelotyping experiments using heart and lung tissue of the G3 generation; color scale as in Figure 2; letters in parentheses denote independent SNPs. Notice the lack of inherited changes from the exposed generation. More groups of fetuses are shown in Additional file 2. This Figure includes standards that are routinely included in the Sequenom runs (see Methods).

spermatozoa (Additional file 4D). We analyzed the methylation patterns of these male germ cells at imprinted DMRs using MIRA-chip. We calculated the average MIRA/input $\log 2$ ratios $(\mathrm{n}=3$ for $\mathrm{MGC}$ and $\mathrm{n}=2$ for sperm) along known imprinted DMRs in the custom imprinting arrays (Figure 7A).

As expected, the paternally methylated DMRs had positive MIRA/input $\log 2$ ratios and the maternally 

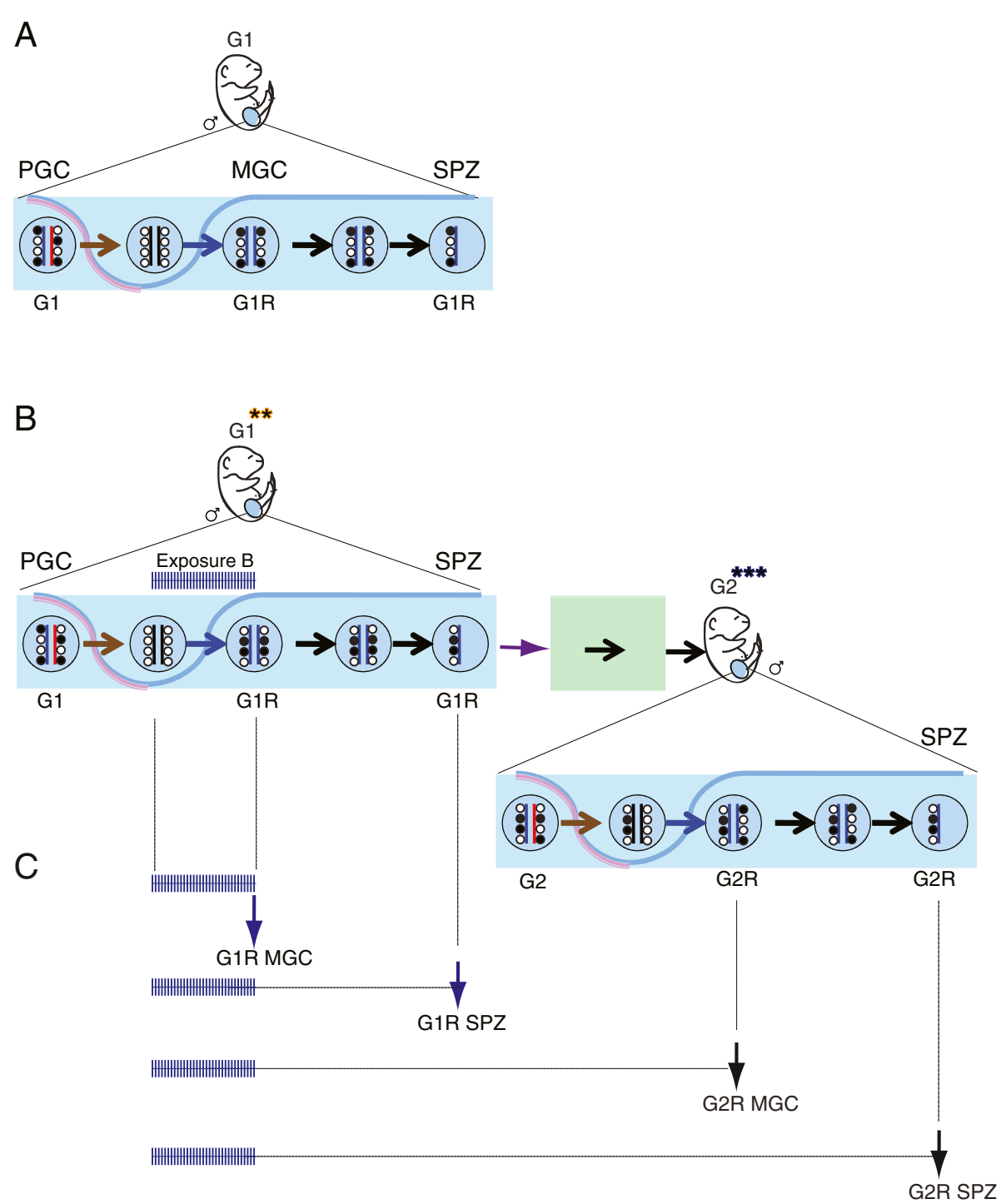

Figure 6 Assessing if in utero exposure to ED results in transgenerationally inherited epigenetic aberration via the germline.

(A) Normal epigenetic remodeling the male germline. MGC undergo normal erasure and normal re-establishment of DNA methylation, producing reprogrammed G1 (G1R) MGCs and G1R spermatozoa. (B) Hypothetical situation where a G1 embryo (two orange stars) exposed to ED during the de novo DNA methylation process results in aberrant reprogramming of G1R MGCs. The aberrant DNA methylation pattern may be maintained in G1R spermatozoa. Aberrant DNA methylation pattern of G1R sperm may harm G2 embryos (three blue stars), by germline epigenetic inheritance. The right panel shows a hypothetical situation in which in the absence of further ED exposure, an aberrant DNA pattern is inherited from the G1R spermatozoa. This aberration fails to be erased in G2 PGCs, and is carried further into G2R prospermatogonia and G2R spermatozoa, which have not been exposed directly or indirectly; thus, persistence of the aberration in these cells would constitute transgenerational epigenetic inheritance. Note that the DNA methylation patterns are simplified, for example, they do not take into account remodeling during the zygote-early embryo stages (green box). (C) Timing scheme of the genome-wide mapping studies. G1 fetuses were exposed in utero during the establishment phase (Exposure B), and G1R and G2R fetal MGCs and adult spermatozoa were collected for analysis.

methylated imprinted DMRs had negative ratios. We calculated the change in MIRA intensities between EDexposed and control G1R prospermatogonia: no MAT DMR showed increased methylation and no PAT DMR showed decreased methylation, using cutoff values of $\pm 5 \%$ and $P<0.05$. We found no changes in G2R-type prospermatogonia at PAT DMRs after any ED treatment
(Additional file 6). There was no detectable change in G2R-type spermatozoa after VZ treatment (Figure 7B), and MAT DMRs were similarly unaffected (Additional file 6). Our data show no evidence for aberrant imprint establishment in propermatogonia after treatment with EDs, for its inheritance through the sperm, or for further transgenerational inheritance to unexposed offspring. 


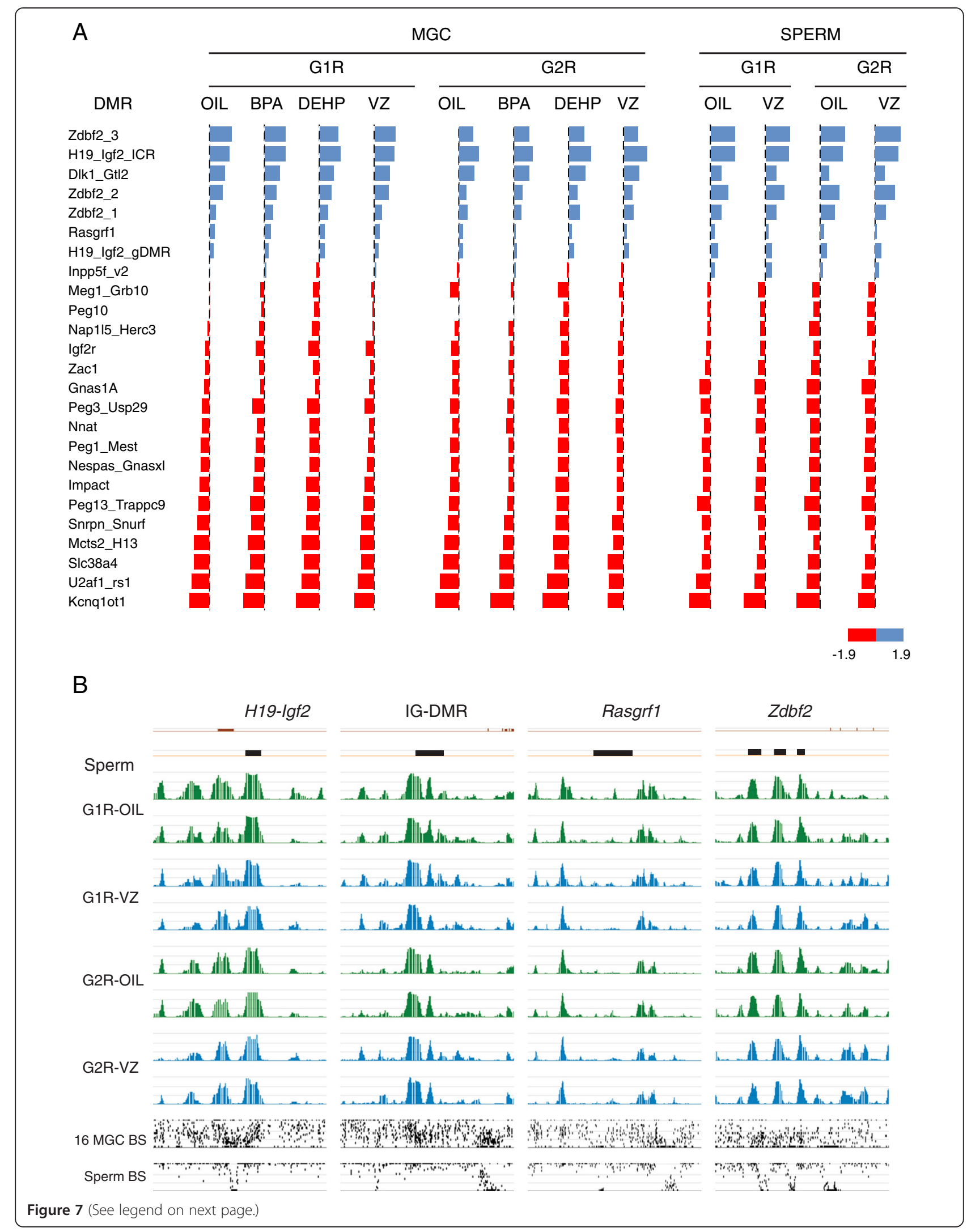


(See figure on previous page.)

Figure 7 DNA methylation establishment is undisturbed by EDs in prospermatogonia at paternally methylated imprinted DMRs. DNA methylation was mapped in purified G1R and G2R prospermatogonia and spermatozoa using MIRA-chip and custom Nimblegen imprinting arrays. The mouse matings were conducted as depicted in Additional file 4. (A) Summary of MIRA-chip results at imprinted DMRs in custom imprinting arrays (groups 5 to 8 ). The average MIRA/input log2 ratios ( $n=3$ for MGCs and $n=2$ for sperm) were calculated for known imprinted DMRs and are depicted with red (maternal and blue (paternal) flags in the range of -1.9 to +1.9 . The full calculations are provided in Additional file 6. Note that paternally methylated DMRs have positive MIRA/input log2 ratios and maternally (MAT) methylated DMRs have negative MIRA/input log2 ratios, as expected. No MAT DMR exhibited increased methylation and no PAT DMR had decreased DNA methylation, using the cutoff values of $\pm 5 \%$ and $P<0.05$ (Student's $t$-test). (B) The MIRA profile is depicted at paternally methylated imprinted DMRs (black rectangles) in biological duplicate samples for VZ treatment or control. The DNA methylation signals of MIRA versus input DNA were plotted as -log $10 P$ values in the range of 0 to 8.4. The average \% DNA methylation levels at each CpG as determined by whole genome bisulfite sequencing (WGBS) are shown compared to that of normal MGCs at $16.5 \mathrm{dpc}$ [18] and normal sperm [20]. Note, that DNA methylation at paternal DMRs is undisturbed by ED treatment in the exposed prospermatogonia and in the prospermatogonia of the next generation.

\section{Search for genome-wide changes in DNA methylation after ED exposure of $\mathrm{G} 1$ prospermatogonia}

To find genome-wide immediate and persistent changes in DNA methylation, we used a genome-wide methylated $\mathrm{CpG}$ island recovery assay (MIRA) with Nimblegen CpG-island + promoter arrays (sample groups 1 to 4 ) and a custom, imprinting array (sample groups 5 to 7 , as summarized in Additional file 7). The custom array included known imprinted genes, DMRs, control genes, IAP-flanking regions, and the Y chromosome. The $\log 2$ ratios of the MIRA/input signal from each hybridization value were quantified, the average values were calculated for the biological replicates, and these values were used in a multi-level data analysis (Table 3 and Additional file 8).

At the first level we tested for changes between pairs of experimental and control samples. We compared the $\log 2$ ratio MIRA versus input values for each sample ' $\mathrm{A}$ ' with the corresponding sample ' $\mathrm{B}$ '. We identified peaks in sample ' $\mathrm{A}$ ' first and calculated the difference between 'A' and 'B' in samples at these locations. We performed this analysis two ways, calling the experimental sample 'A' and control sample 'B' and vice versa, to ensure that a change would be detected at each peak even when it occurred only in 'A' or in 'B'. We chose very low cutoff values - changes greater than $\pm 5 \%$ with Fisher's exact test $P$ value $(P<0.05)$ - to allow the higher-level analyses of a larger set of primary hits. At the second analysis level, we compared the results of two level-1 tests to find common changes between G1R and G2R samples. At level 3, we compared the results of two level-1 tests to find common changes between MGCs and sperm in the same generation. At level 4, we compared the results of four level-1 tests: an immediate effect in G1R MGCs that persisted into G1R sperm, G2R prospermatogonia, and G2R sperm, thereby having the potential to affect the G3 soma.

We found very few hits at levels 2 to 3 and no hits at level 4, despite the low cutoff values (Table 3 and Additional file 8). Some examples of the best hits of the level 2 and 3 analyses are shown in Figure 8A; these hits are unimpressive and exist at regions with generally low DNA methylation.

We inspected the locations of the best hits in a recent study of G3R sperm after initial VZ exposure in G1 MGCs [43]. We found no change in G1R and G2R MGCs and sperm at Mro and found a complete lack of DNA methylation at Elf3 (Additional file 9). One possible explanation for the discrepancies can be methodological differences. Whereas we hybridized each biological replicate against input and measured methylation levels along chromosomes, Guerrero-Bosagna hybridized control and experimental samples against each other, measuring differences in each chip, thus lacking information about DNA methylation levels at specific regions.

\section{Search for changes in DNA methylation at IAPs}

Interesting targets for ED-caused aberrant DNA methylation may be intracisternal A-particle elements (IAPs), which retain substantial DNA methylation through the germline erasure process and thus can lead to transgenerational inheritance (TGI) of epigenetic changes $[18,44]$. Because most IAPs become highly methylated by the spermatozoa stage, it may be the rare unmethylated IAPs that carry specific information to the respective transcripts they may affect, and in turn, these may depend on the protection by H3K4 methylation [19]. We calculated the average DNA methylation along each unique 1-kb-long IAP-flanking region in the custom imprinting arrays and identified the DNA methylation changes at these regions between the exposed and control-treated samples with cutoff values of $\pm 5 \%$ and $P<0.05$ (Additional file 10).

To reveal TGI of DNA methylation aberrations, we searched for common changes at IAP-flank regions between G1R and G2R in MGC samples after the initial exposure of G1R MGCs to VZ; those changes are shown with chromosomal coordinates in Additional file 11. Similarly, we identified the changes at IAP-flanking regions that were common between VZ-exposed G1R prospermatogonia and G1R sperm or between G1R and 
Table 3 Analysis results for immediate and persistent changes in DNA methylation after ED exposure of G1 prospermatogonia

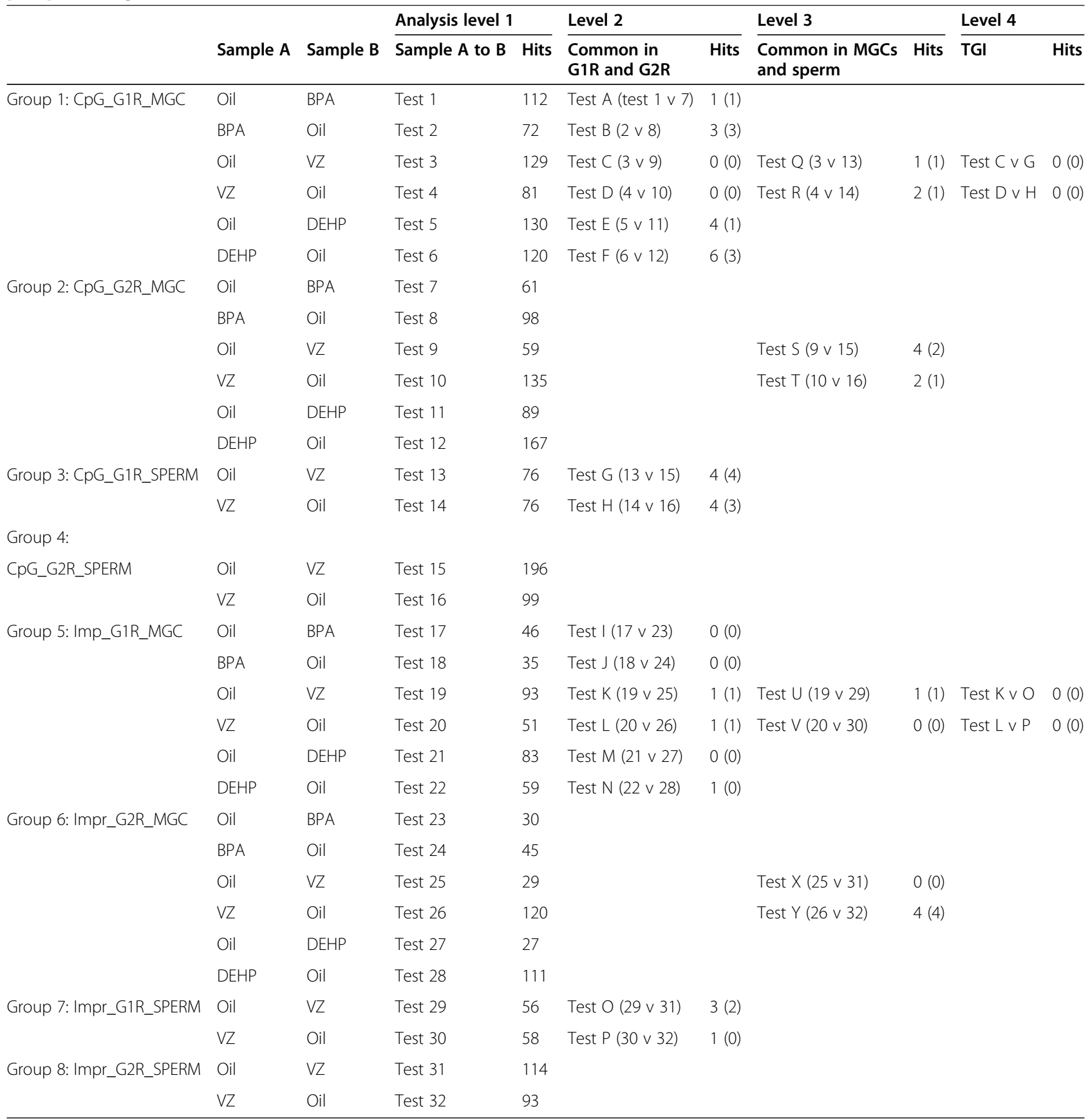

After in utero exposure of G1 MGC to vehicle control ('oil') or one of the EDs, the level of DNA methylation was measured in reprogrammed G1R and G2R fetal MGCs and adult spermatozoa by MIRA-chip and Nimblegen microarrays. CpG-promoter arrays were used in groups 1 to 4 and custom imprinting arrays were used in groups 5 to 7 . At the first level of analysis, we performed 32 tests comparing the log 2 ratio MIRA versus input values. To detect a change between sample $A$ and $B$, peaks were identified in the average value ( $n=3$ for MGC and $n=2$ for sperm) of $A$ samples first and were compared with the MIRA intensity of the average of sample B at the same locations. The number of hits where a change occurred with greater than $\pm 5 \%$ and Fisher's exact test $P$ value $(P<0.05)$ were tabulated. At level two, we compared the results of two level-1 tests (in parentheses) to find common changes between G1R and G2R in MGCs (Tests A-F and I-N) and in sperm (Tests G-H and O-P); the number of common hits is provided and those that changed in the same direction are given in parentheses. At level three, we compared the results of two level-1 tests to find common changes between MGC and sperm in the same generation, G1R (Test Q-R and U-V) or G2R (S-T and $X-Y)$. At level four, we compared the results of four level-1 tests in search for true transgenerational epigenetic inheritance: immediate effect in G1R MGCs, maintained into G1R sperm, G2R prospermatogonia, and G2R sperm, thereby having the potential to affect G3 soma. Note the low number of hits in levels 1 to 3 and lack of hits at level 4 , even at the low cutoff values applied. 


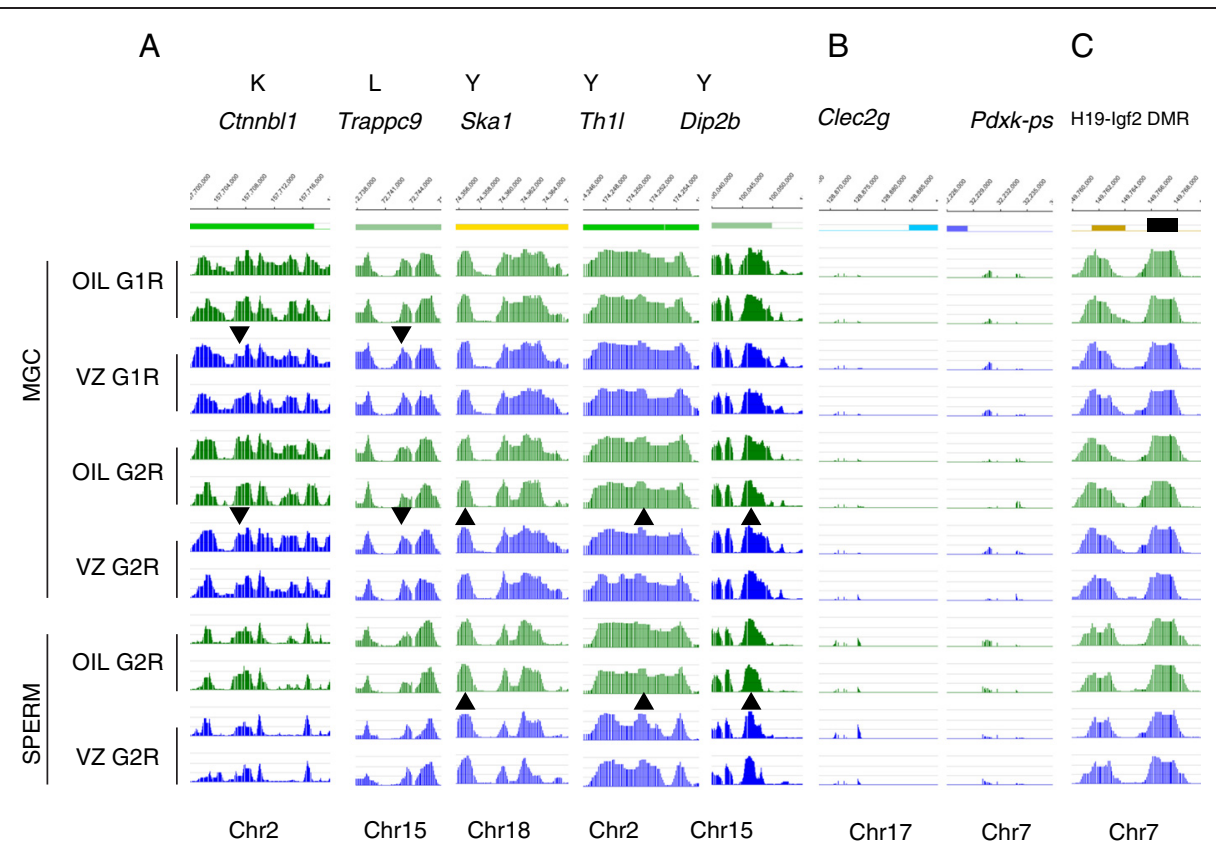

Figure 8 Selected top hits of transgenerationally inherited DNA methylation aberrations. Prospermatogonia of G1 fetuses were treated with ED or oil control in utero as depicted in Additional file 4. Next, DNA methylation was mapped using MIRA-chip and custom Nimblegen arrays in purified G1R and G2R prospermatogonia at $17.5 \mathrm{dpc}$ in triplicate and in adult spermatozoa in duplicate. Immediate and persistent changes are tabulated in Table 3 and Additional file 8. (A) Selected top persistent hits are shown from the analysis in duplicates labeled at the top according to the comparisons in Table 3 and marked with arrowheads (up for increase and down for decrease) (B) A selected IAP-flank region where common changes were detected in MGC samples between G1R and G2R at and between G2R MGC and G2R sperm. (C) The H19-lgf2 imprinted DMR is shown as a positive control for the DNA methylation signal (black rectangle). DNA methylation signals of MIRA versus input DNA are plotted as -log $10 P$ values ranging from 0 to 8.3 for experimental and control replicate samples. Note that these top changes are minor and not highly significant.

G2R sperm (Additional file 11 and Figure 8B). We also identified the common changes in G1R and G2R prospermatogonia that occurred after initial DEHP or BPA exposure in G1R (Additional file 10). The changes were small, often occurred in the opposite direction, and seldom mapped to gene promoters.

Our MIRA-chip results collectively suggest that BPA, DEHP, and VZ at the given doses have negligible immediate and persistent effects on the de novo DNA methylation process in mouse G1R prospermatogonia at $\mathrm{CpG}$ islands, promoters, imprinted DMRs, IAPs, and along the $\mathrm{Y}$ chromosome.

\section{Search for immediate and persistent changes in genome-wide transcription after ED exposure of G1 prospermatogonia}

Although we found no evidence for TGI at the level of DNA methylation, other mechanisms such as histone modifications, histone variants, and long non-coding RNAs also participate in gene regulation and may transmit epigenetic aberrations between generations. Such aberrations are likely manifest in altered gene expression patterns. Therefore, we carried out Affymetrix microarray hybridization experiments using RNA from FACS-sorted 17.5-dpc fetal FGCs and MGCs exposed in utero to BPA, DEHP, VZ, or vehicle control. To find immediate direct responses to ED exposure, we analyzed G1R fetal oocytes and prospermatogonia. To find persistent changes, we analyzed $G 2 R$ prospermatogonia. For the summary of samples, see Additional file 7.

We found that sex was the main dividing parameter in principal component analysis (PCA) (Figure 9A), as expected based on our previous transcription profiling of fetal germ cells using RNA-seq [19]. We confirmed the male or female germ cell-specific transcription of epigenetic modifiers: Mll3 and Ehmt2 were highly expressed in FGCs and MGCs, respectively, and neither showed any change in transcription in response to any of the EDs (Figure 9B). G1R and G2R male germ cells were slightly separated from each other in the PCA (Figure 9A), likely because these samples were run at different times or because of the reciprocal genome composition $(\mathrm{FVB} \times \mathrm{OG} 2$ and $\mathrm{OG} 2 \times \mathrm{FVB}$, respectively) of these cells. Importantly, G1R prospermatogonia samples were clustered based on treatment type: BPA samples shifted slightly from control oil samples, and groups of DEHP and VZ samples shifted further away. Such clustering of samples was not apparent in G1R female germ cells or G2R male germ cells (Figure 9A). Using sex as an internal biological difference, we compared G1R female 
A

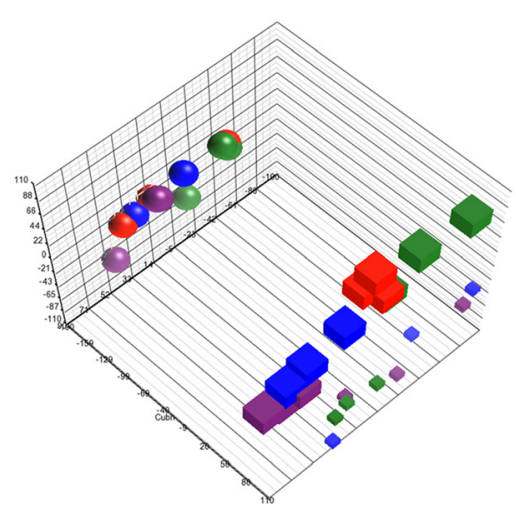

C

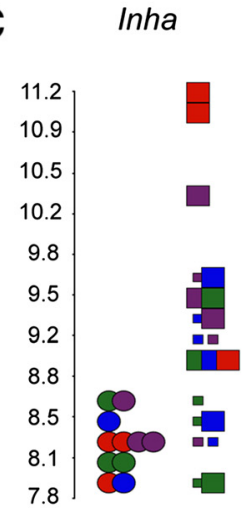

$\mathrm{D}$

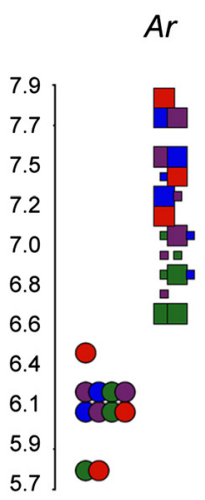

Prss35
B

MII3

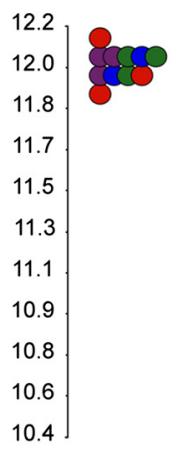

Ehmt2
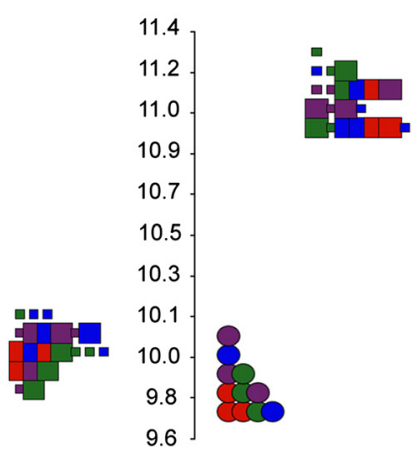

Gja1

Amh
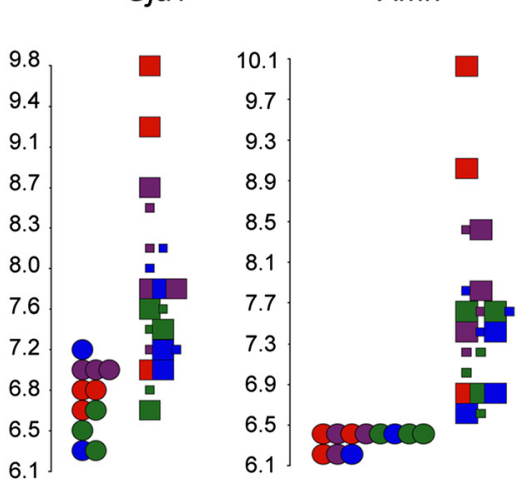

Hsd17b3

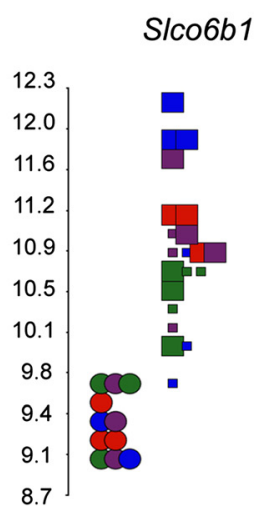

Figure 9 Search for immediate and persistent changes in RNA levels after ED exposure of G1 prospermatogonia. G1R prospermatogonia were purified at $17.5 \mathrm{dpc}$ from female or male fetuses exposed in utero to BPA, DEHP, VZ, or vehicle control ('oil') and G2R prospermatogonia were also purified. Trancription of mRNA was measured using Affymetrix 1.0ST chips and the data were statistically analyzed in the Partek suite. (A) Principal component analysis of the samples. Note that the major principal component is sex and the second is generation. In addition, G1R male samples are separated by ED treatment. (B) Selected control transcripts are shown with known reciprocal expression patterns in the two sexes. (C) Selected top hits are shown that are upregulated in BPA-exposed G1R prospermatogonia. These genes are known targets of $\beta$-estradiol. (D) Selected top hits in VZ- and DEHP-exposed G1R propsermatogonia. Note that the androgen pathway is affected.

control oil samples with G1R male controls and found 11,848 statistically significant differences $(6,552$ up in female, 5,296 up in male) between sexes using 1.5-fold change and a false discovery rate (FDR) of 0.05 (Table 4). Similar numbers were found when comparing the respective female and male samples after BPA, DEHP, and VZ treatment. Notably, we found no overlap in the female up hits in the controls with the female down hits in any of the samples. These comparisons assured us that the data quality and analysis pipeline 
Table 4 Search for immediate and persistent changes in RNA transcript levels after ED exposure of G1 prospermatogonia

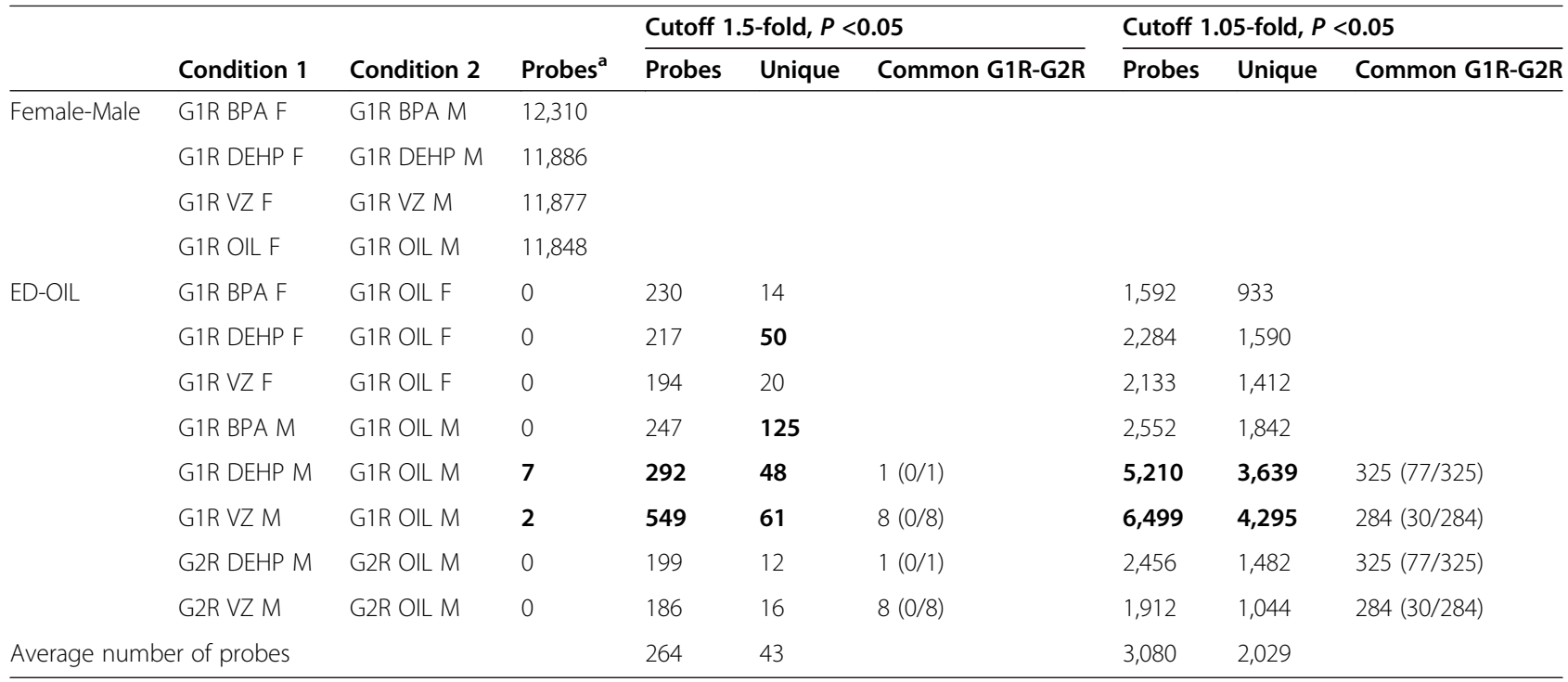

${ }^{a}$ Cutoff: 1.5 -fold, FDR $P<0.05$.

Prospermatogonia were purified at $17.5 \mathrm{dpc}$ from female (F) or male (M) fetuses exposed in utero (G1R) to BPA, DEHP, VZ, or vehicle control ('oil'). Prospermatogonia were also purified from the next generation (G2R). Trancription of mRNA was measured using Affymetrix 1.0ST chips. Transcription differences were detected between conditions 1 and 2 . The hits are tabulated according the cutoff values, shown in the heading, as probes and unique transcripts. Bold numbers are higher than average among ED treatments for the given cutoff values. Common changes were detected between the unique transcripts that change in G1R and G2R samples for the same treatment; the numbers of those that changed in the same direction are in parentheses.

allows us to detect inherent biological differences in this sample set.

We generated the counts of differentially expressed probes and unique transcripts using Ingenuity Pathway Analysis (IPA) for each ED exposure versus control (Table 4). Using cutoff values of 1.5 -fold change and FDR $P<0.05$, we found seven probes and two probes in the G1R DEHP male and G1R VZ male samples, respectively, but no probes in the other samples. When we relaxed the statistical cutoff value to simply $P$ value $<0.05$ (with 1.5-fold difference), we found an average of 264 probes per condition; using cutoffs of 1.05 -fold and $P$ value $=0.05$, we found an average of 3,080 probes per condition. We considered these relaxed cutoff values rather loose and likely to result in false positives. However, at any cutoff value, G1R DEHP versus control male and G1R VZ versus control male samples consistently yielded larger numbers of differences than the average numbers of other comparisons. In addition, the number of unique transcripts was higher than average in G1R DEHP versus control female and G1R BPA versus control male samples. This suggested that there are subtle transcription changes in these four conditions that are caused by ED exposure. IPA analysis revealed that the transcription changes affected the reproductive or endocrine system in each case. Interestingly, IPA identified $\beta$-estradiol as the upstream regulator for G1R BPA MGCs, with a probability of $4.57 \times 10^{-19}$; four transcripts of the top 10 are regulated by $\beta$-estradiol (Figure $9 \mathrm{C}$ ). Selected top hits in the male
G1R VZ and DEHP samples are depicted in Figure 9D. Hydroxysteroid (17-beta) dehydrogenase 3 (Hsd17b3) was upregulated by BPA and VZ, while androgen receptor $(A r)$ was upregulated by VZ and DEHP. These transcripts are interesting because they play roles in the androgen pathways.

We noticed that none of the G1R top changes were detected in G2R (Figure 9). To further search for persistent changes in transcription, we compared the lists of differential expression in G1R MGCs with the respective G2R samples (Table 4). At the cutoff values of 1.5 -fold and $P<0.05$, we found only one and eight common changes for DEHP and VZ, respectively, but none of these unique transcripts changed in the same direction. At the cutoff values of 1.05 -fold and $P<0.05$, we found 325 and 284 common changes for DEHP and VZ, respectively. However, only $77 / 325$ and $30 / 284$ unique transcripts changed in the same direction, fewer than expected by chance (Additional file 12). When we considered all probes with common changes between G1R and G2R, Fisher's exact tests revealed that a significantly greater number of the common changes occurred in the opposite direction. For DEHP, G0 downregulated (G1:Down) genes are significantly more likely to be in G1:Up $(1.8 \times$, $P=2.6 \times 10^{-5}$ ), and G0:Up genes are significantly more likely to be in G1:Down $\left(4.0 \times, P<2.2 \times 10^{-16}\right)$. For VZ, G0: Down genes are significantly more likely to be in G1:Up $\left(5.4 \times, P<2.2 \times 10^{-16}\right)$, and G0:Up genes are significantly more likely to be in G1: Down $\left(7.2 \times, P<2.2 \times 10^{-16}\right)$. 
In summary, ED exposures caused relatively small effects compared with those resulting from sex and generation number. The changes, however, affected hormonal pathways for the G1R BPA, G1R DEHP, and G1R VZ male samples, confirming that the drugs have reached their targets in fetal germ cells. The ED treatments had more effect in prospermatogonia than in fetal oocytes, likely because male germ cells undergo epigenetic establishment phase at the fetal stages and the establishment process may be vulnerable to environmental disturbances. VZ and DEHP caused more transcription differences in male germ cells than BPA, possibly because male germ cells may be more responsive to androgen signaling than to estrogen signaling. Notably, no treatment effect on transcription persisted from G1R to G2R, suggesting that the germ line is capable of rebounding from epigenetic effects caused by EDs.

\section{Discussion}

To date no molecular evidence exists in mammals that fulfills the following criteria of TGI after in utero exposure: (1) an epigenetic aberration is detected in the exposed fetal germ cells; (2) the aberration is retained in the gamete; and (3) the same aberration is detected in the germ cells of the next generation. The aim of the present study was to systematically and rigorously evaluate the effects of EDs on global epigenetic reprogramming in the male mouse germ line after in utero exposure. We selected EDs that were reported to cause epigenetic aberrations, and focused on three EDs that affect estrogenic and androgenic pathways. Indeed, our exposures have reached the fetal germ cells, as we detected specific changes of transcription in G1R MGC that could be expected based on the known estrogenic properties of BPA and anti-androgenic properties of DEHP and VZ. We found that BPA caused the activation of estrogen-responsive genes, whereas VZ and DEHP induced elevated $H s d 17 b 3$ and $A r$ transcripts, respectively, in the exposed G1R MGCs. However, these changes did not persist into the G2R MGCs. We investigated global DNA methylation changes at $\mathrm{CpG}$ islands, promoters, imprinted DMRs, and IAP repeats, and we did not find evidence for persistent changes between G1R and G2R prospermatogonia.

Even if we encountered a very persistent epigenetic aberration that occurred in the fetal germline of one sex and was maintained through several generations, we would expect to find a dilution of this effect with every generation, because meiosis results in haploid gametes and the chance of getting this allele is halved in every consecutive generation. The only exemption to this rule would involve the Y chromosome. We found no persistent changes in global transcription or DNA methylation between the exposed G1R and the next G2R generations along the Y chromosome.
TGI is perhaps easier to explain in organisms like $C$. elegans, where the germline is set aside at the zygote stage [45]. Even though the C. elegans germline also undergoes global epigenetic remodeling that mainly involves erasure and re-establishment of active histone modifications [46], any epigenetic aberration could be more easily inherited in the daughter cells that remain in the germ lineage. Indeed, in C. elegans, deficiencies in the H3K4me3 chromatin modifiers in the parental generation extended the life span of three generations in the wild type descendants [47]. However, in mammals, the germline develops from progenitors in the proximal epiblast and these cells have already differentiated away from the pluripotent state. Any epigenetic aberration has to resist two global waves of epigenetic reprogramming: the first occurs after fertilization in the zygote-preimplantation stage and the second in the primordial germ cells. Both waves involve erasure of the old patterns and the reestablishment of new patterns. These two global waves of remodeling must be the mechanism that removes epigenetic damage caused by the environment, ensuring that these are not inherited into the soma of the G3 generation. It is tempting to speculate whether avoiding TGI of environmental aberrations played an evolutionary role in the development of dual global reprogramming events in mammals.

\section{Conclusions}

Our data show that whereas endocrine disruptors affect the transcription and DNA methylation state of exposed germ cells, these changes are not found in the germ cells of the subsequent generation. The genome-wide epigenetic remodeling processes in the next generation are robust, allowing the mammalian germline epigenome to recover from the effects of in utero exposure to endocrine-disrupting chemicals.

\section{Methods}

\section{Ethics statement}

Housing and care of the animals were consistent with Public Health Service Policy, the NIH Guide for the Care and Use of Laboratory Animals, and the Animal Welfare Act. All of the animal experiments were approved under protocol ID 91023 by the IACUC, City of Hope.

\section{Treatment regimens for the selected EDs}

Mouse transgenic line TgOG2 [28] and inbred FVB, 129S1, and JF1 mice were used in the various studies. Animals were housed in polypropylene cages and received a special verified diet, 5 K96 (TestDiet), as recommended by the NIH for animal studies involving hormone-like chemicals. Drinking water was provided in glass bottles and was purified on a carbon filter (Filter Cartridge Hi-Cap Carbon 9-3/4 ID \#: 2100-1970-102 from Edstrom Direct) 
just upstream of the bottle filler. Pregnant females in generation 0 (G0) were gavaged with EDs daily for 5 days starting at $8.5 \mathrm{dpc}$ for the erasure study or at $12.5 \mathrm{dpc}$ for the establishment study.

The EDs used were vinclozolin (ChemService Catalog no. PS-1049; Sigma, USA), bis(2-ethylhexyl) phthalate (Selectophore, (DEHP), Catalog no. 80030; Fluka/Sigma Inc.), and bisphenol A (Catalog no. 239658; Sigma Aldrich Inc.). All three EDs were dissolved/suspended in tocopherol-stripped corn oil vehicle (MPI Catalog no. 0290141584). Control animals were treated with the oil vehicle alone $[48,49]$. The oral doses for VZ (100 $\mathrm{mg} / \mathrm{kg} /$ day $)$, BPA $(0.2 \mathrm{mg} / \mathrm{kg} /$ day $)$, and DEHP (750 $\mathrm{mg} / \mathrm{kg} /$ day) were the same as in our previous study [32]. These doses to pregnant mice are known to reach and to affect the fetus. VZ, for example, given by oral gavage at 10 or $50 \mathrm{mg} / \mathrm{kg}$ doses daily between $13.5 \mathrm{dpc}$ and $17.5 \mathrm{dpc}$ resulted in morphological changes in mouse fetuses at $19.5 \mathrm{dpc}$, including feminization of males (hypospadias) and virilization (longer urethras) of females together with altered gene expression in the genital tubercles [50,51]. Even a low dose of $1 \mathrm{mg} / \mathrm{kg}, \mathrm{VZ}$ administered to pregnant mice in drinking water between 15 and $22 \mathrm{dpc}$ resulted in decreased anogenital distance, prostate weight, relative testis weight, sperm count, and acrosomal state; caused increased sperm head abnormalities and pathology of the testes; and affected the expression of selected genes in male G1 offspring [52]. In utero and lactational exposure to DEHP at $750 \mathrm{mg} / \mathrm{kg} /$ day caused severe male reproductive system toxicity in rats including reduced sperm count and testicular malformations [48]. In mice, DEHP administration to pregnant mouse dams in food at $0.01 \%$ or $0.03 \%$ [53] (an estimated 0.75 to $1 \mathrm{~g} / \mathrm{kg}$ or 2.25 to $3 \mathrm{~g} / \mathrm{kg} /$ day dose, respectively), caused fetal defects and reduced the number of litters and number of live pups. At such daily doses, the DEHP metabolite MEHP clearly showed dose-dependent accumulation in 18.5-dpc fetal livers [54]. Oral administration of a single dose of $10 \mathrm{mg} / \mathrm{kg}$ BPA to pregnant mouse dams resulted in $0.01 \mathrm{mg} / \mathrm{L}$ $\mathrm{BPA}$ or $0.03 \mathrm{mg} / \mathrm{L}$ total BPA (including metabolite) in 15.5-dpc fetuses in the first hour [55], and three consecutive daily doses were additive. Feeding BPA $(0.02 \mathrm{mg} / \mathrm{kg})$ to pregnant mice at 11.5 to $17.5 \mathrm{dpc}$ significantly decreased sperm production in the male offspring [8] and disturbed oocyte development and meiosis in female fetuses [56]. The same dose of BPA at 11.5 to $14.5 \mathrm{dpc}$ lead to a large number of subtle changes of transcription in the fetal ovary [57].

\section{Purification of germ cells}

Using a MoFlo or Aria III flow cytometer, germ cells (GFP-positive), and somatic cells (GFP-negative) were flow-sorted from embryonic or fetal gonads at 9.5, 13.5, or $17.5 \mathrm{dpc}$ based on germ cell-specific EGFP expression in the TgOG2 transgenic mouse line [28] as described previously [58]. Phenol red was excluded from the M2 medium. Spermatozoa were collected from the cauda epididymis of adult FVB males and the motile fraction was used for MIRA-chip analysis.

\section{DNA isolation and methylated $\mathrm{CpG}$ island recovery assay (MIRA) and MIRA-chip}

Genomic DNA was isolated from fetal germ cells by proteinase $\mathrm{K}$ digestion and phenol-chloroform extraction. Contaminating RNA was removed by RNAse treatment (Roche). RNA-free genomic DNA was sonicated to 300 to $800 \mathrm{bp}$ using a standard Bioruptor water bath sonicator (Diagenode). Sonicated DNA (500 ng) was used for MIRA, as described previously [40]. The methylated fraction was captured using recombinant MBD2b and MBD3L1 proteins as described earlier [59] and was amplified by ligation-mediated $\mathrm{PCR}$ as previously described [42]. CpG-promoter arrays and custom-designed tiling arrays (110228_MM9_PS_ChIP), including all known imprinted domains and IAP flanking regions (Roche/ NimbleGen), were used for the CpG methylation profile analysis [19]. Amplified MIRA DNA fractions were compared with amplified input DNA. Data were extracted from scanned images by using NimbleScan 2.3 extraction software (NimbleGen Systems).

\section{Detecting DNA methylation changes in MIRA-chip}

To identify statistically significant changes caused by ED treatment, we compared treated versus oil control and also oil control versus treated samples in triplicates. To identify individual peaks in sample A, probes were considered positive if their normalized $\log 2$ ratio was above the 95th percentile of all probes on the array, and peaks were defined as four or more consecutive positive probes allowing one gap. The common peaks were identified between the triplicates of sample A and their mean value was compared to that of sample B. DNA methylation changes were identified based on the average $\log 2$ ratio signal difference between sample A and sample B, using cutoff values of $\pm 5 \%$ (minimum 1.05 -fold increase or 0.95 -fold decrease) and Fisher's exact $t$-test $P$ value, $P<0.05$. To compare DNA methylation levels at DMRs and IAPs, we calculated the average $\log 2$ MIRA/input values along these sequences and compared them to detect changes between conditions with the cutoff values of $\pm 5 \%$ and $P<0.05$ (Student's $t$-test).

\section{RNA isolation and Affymetrix microarray hybridization}

RNA was isolated from germ cells using TRIzol (Qiagen) extraction followed by ethanol precipitation. The RNA samples were processed in the City of Hope's Microarray Facility using Mouse Gene 1.0 ST arrays (Affymetrix). 
Fold-change values were calculated based upon the least-squares mean using Partek Genomics Suite. Prior to statistical analysis, data were normalized using robust multichip average (RMA) normalization [60]. We used an ANOVA model with linear contrast to identify genes that have a change under condition $\mathrm{A}$ relative to condition $\mathrm{B}$, with specific statistical significance and fold-change values, as specified in the text. We considered interactions between treatment, sex, and generation. Microarray data were deposited in GEO: Super series GSE59543.

\section{RNA isolation and reverse transcription-PCR}

RNA was isolated from germ cells and various organs/ body parts using RNA-Bee (Tel-Test). Contaminating DNA was removed with the DNA-free Kit (Ambion). cDNA was reverse-transcribed from total RNA using SuperScript III First-Strand Synthesis kit (Invitrogen).

\section{Analysis of allele-specific DNA methylation and gene expression by Sequenom allelotyping}

Allele-specific DNA methylation and gene expression was measured by multiplex SNuPE assays $[29,61]$ on the Sequenom platform, as we have done previously $[32,41,62,63]$. These assays are based on single nucleotide polymorphisms (SNPs) that distinguish between the inbred JF1/Ms (JF1) and 129S1 (129) mouse strains, or between the JF1 and the TgOG2 (OG2) transgenic mouse strains. Each SNuPE primer (UEP) abuts a SNP in a target DMR/transcript, and the incorporating nucleotides differ in molecular mass between the parental alleles. The abundance of the extended UEP is quantified by mass spectrometry. MIRA-enriched samples or amplified cDNA samples were spotted onto a 384 SpectroCHIP Array. Automated spectra acquisition was performed in a MassArray Compact mass spectrometer (Sequenom) using the Spectroacquire program (Sequenom) and was analyzed by MassArray Typer v3.4. RNA-mixing standards were routinely run to verify linear response in measured versus input allele-specific transcription: for example, total RNA from JF1 and 129 embryos was mixed in different percent ratios $(0: 100,10: 90,30: 70,50: 50$, 70:30, 90:10, and 100:0) before cDNA preparation and Sequenom allelotyping. A true heterozygote DNA sample was used for DNA skew correction; a 50:50 RNA mix was used for RNA skew correction. The percentage of DNA methylation or transcription of each allele in the total methylation or expression was calculated at each given SNP. Primers are listed in Additional file 13.

\section{Statistical analysis}

All statistical test $P$ values refer to Student's $t$-test unless otherwise noted.

\section{Additional files}

Additional file 1: Sequenom data tables. (A) Testing the effect of endocrine-disrupting chemicals (EDs) on imprint erasure. The full dataset depicted in Figure 2 is provided. (B) Testing the effect of EDs on the imprint establishment in prospermatogonia by measuring allele-specific DNA methylation at DMRs in G2 soma. The full dataset depicted in Figure $3 \mathrm{C}$ is provided. (C) Testing the effect of EDs in fetal primary oocytes by measuring allele-specific DNA methylation at DMRs in G2 soma. The full dataset depicted in Figure 3D is provided. (D) Effect of EDs on allele-specific expression of imprinted genes in G2 embryos derived from exposed prospermatogonia. The full dataset depicted in Figure 4 is provided. (E) Effect of EDs on allele-specific expression of imprinted genes in larger number of $\mathrm{G} 2$ embryos derived from exposed prospermatogonia. The full dataset depicted in Additional file 2, panel A is provided. (F) Testing for transgenerational epigenetic inheritance of aberrant imprinted expression in G3 soma. The full dataset depicted in Figure 5 is provided. (G) Testing for transgenerational epigenetic inheritance of aberrant imprinted expression in larger number of G3 fetuses. The full dataset depicted in Additional file 2, panel B is provided. $\mathbf{( H )}$ Effect of EDs on allele-specific expression of imprinted genes in embryos derived from exposed primary oocytes. The full dataset depicted in Additional file 3 is provided.

Additional file 2: Analysis of larger number of fetuses. (A) Effect of EDs on allele-specific expression of imprinted genes in fetuses derived from exposed prospermatogonia. Average parental allele-specific transcription in different body parts/organs of three $13.5 \mathrm{dpc}$ G2 fetuses is displayed. The experiment was conducted as depicted in Figure 3A. Results of RNA Sequenom allelotyping experiments of selected imprinted transcripts listed to the left are shown using the color scale as in Figure 2. There were no statistically significant ( $P$ value $<0.05$ ) differences between $E D$ and oil control, greater than 5\%. The number (n) of G2 fetuses whose average values are shown per each ED is indicated. (B) Testing for transgenerational epigenetic inheritance of the aberrant imprinted expression. Average parental allele-specific transcription in different body parts/organs of three 13.5 dpc G3 fetuses is displayed. The experiment was performed as described in Figure $5 \mathrm{~A}$ and displayed according to the color scale in Figure $2 \mathrm{~B}$. There were no statistically significant ( $t$-test, $P<0.05$ ) differences between ED and oil control, greater than 5\%. The number of G2 fetuses whose average values are shown per each ED is indicated.

Additional file 3: Effect of EDs on allele-specific expression of imprinted genes in embryos derived from exposed primary oocytes. The experiment was conducted as depicted in Figure 3B. Parental allele-specific transcription is displayed according to the color scale as in Figure 2. Results of RNA Sequenom allelotyping experiments of imprinted transcripts listed to the left are shown in different body parts/organs of the 13.5 dpc G2 embryo after ED or oil treatment of the G0 dam. Statistically significant $(P<0.05)$ differences as compared to oil control greater than $5 \%$ and $10 \%$ are indicated by thin or bold rectangles, respectively. Note the strict undisturbed allele-specific transcription. Notice that there is no causative relationship between aberrant allele-specific DMR methylation (Figure 3D) and aberrant allele-specific transcription in this Figure.

Additional file 4: Experimental designs for testing the effect of EDs on the establishment and maintenance of DNA methylation in the male germ line and for transgenerational inheritance of epigenetic aberrations. (A, B) Experimental design to directly test the effect of ED exposure on paternal DNA methylation establishment in the offspring. G1 male offspring of FVB dam and OG2 father was exposed in utero at the time when paternal imprint establishment occurs in its prospermatogonia and MAT DMRs are protected from de novo DNA methylation. Exposure occurred daily from $12.5 \mathrm{dpc}$ to $16.5 \mathrm{dpc}$ by oral gavage to pregnant $\mathrm{G} 0$ dams with one of the three different EDs or vehicle control (oil). (A) Prospermatogonia were collected at $17.5 \mathrm{dpc}$ for DNA methylation analysis from G1 fetuses by FACS sorting. In these prospermatognia, G1-specific DNA methylation is erased, and DNA methylation re-establishment is largely complete, resulting in reprogrammed G1 (G1R) pattern. (B) After reaching adulthood, G1R spermatozoa (developed from in utero exposed prospermatogonia) were also collected from G1 (FVBXOG2) males (green testicles). (C, D) Experimental design to test if perturbing DNA methylation establishment in prospermatogonia is transgenerationally inherited through 
the paternal germ line to an unexposed generation. G1 male offspring of FVB dam and FVB father was exposed in utero at the time when paternal imprint establishment occurs in its prospermatogonia and MAT DMRs are protected from de novo DNA methylation. Exposure occurred daily from $12.5 \mathrm{dpc}$ to $16.5 \mathrm{dpc}$ by oral gavage to pregnant $\mathrm{G} 0$ dams with one of the three different EDs or control oil vehicle. After reaching adulthood G1 male was mated with OG2 females and from G2 fetuses (derivative of exposed prospermatogonia, marked by three blue stars) prospermatogonia (never exposed to EDs) were collected for DNA methylation analysis by FACS sorting. These spermatogonia carried the reprogrammed G2 (G2R) DNA methylation pattern. (D) Some G2 (FVBXOG2) males were allowed to reach adulthood and were used for collecting G2R-type spermatozoa (developed from never exposed prospermatogonia).

Additional file 5: MIRA-chip profile of imprinted DMRs in G1R and G2R prospermatogonia. DNA methylation was mapped using MIRA-chip and custom Nimblegen arrays in prospermatogonia purified by FACS. The MIRA profile is depicted in the neighborhood of paternally (page 1) and maternally (page 2) methylated imprinted DMRs (black arrowheads) in biological triplicate samples for each treatment as indicated on the side. The DNA methylation signals of MIRA versus input DNA were plotted as - $\log 10 P$ value scores ranging from 0 to 8.4 for gestational stage $17.5 \mathrm{dpc}$. The experiment was conducted as depicted in Figure 4. Note, that in prospermatogonia, default establishment of DNA methylation is undisturbed at paternally methylated DMRS and the protection from DNA methylation establishment is also undisturbed at maternally methylated imprinted DMRs.

Additional file 6: No transgenerational epigenetic aberrations are detectable at imprinted DMRs after in utero ED exposure. Samples (groups 5 to 8) were analyzed from the MIRA-chip experiment conducted using the custom imprinting arrays (Additional file 7). The average MIRA/ input $\log 2$ ratios ( $n=3$ for $M G C$ and $n=2$ for sperm) were measured along known imprinted DMRs (column A), at the chromosomal locations indicated (columns B to D). The average values of two conditions were compared to detect changes along these DMRs with the cutoff values of $\pm 5 \%$ and $P<0.05$. In the following columns the MIRA/input log 2 ratios of each individual sample are also provided together with standard deviation values of the Student's $t$-test.

\section{Additional file 7: List of MIRA-chip and Affymetrix samples.}

\section{Additional file 8: Results of MIRA-chip experiments.}

Prospermatogonia of G1 fetuses were treated with ED or oil control in utero as depicted in Additional file 4. DNA methylation was measured in purified G1R and G2R prospermatogonia (MGC) at $17.5 \mathrm{dpc}$ and in adult spermatozoa by MIRA-chip. We used CpG-promoter or custom imprinting Nimblegen arrays. The analysis was conducted at four levels, as described in the legend to Table 3. In addition, at level 5, we compared the hits of level 1 to 4 analysis between the two types of arrays. Each peak (chromosomal coordinates are given in columns $\mathrm{F}$ to $\mathrm{H}$ ) belongs to a level 1 test (column C) and may belong to a level 2 to 4 tests (column D) between two samples or two to four level 1 tests (column E). The MIRA intensities in each of the replicate and the average $A$ and $B$ samples are provided as log2 MIRA/input ratios (columns I to P). The differences in log2 MIRA/input ratios are given in column $\mathrm{Q}$ with color codes (red increase in A versus $\mathrm{B}$, blue, decrease in $A$ versus $B$ ) with $t$-test $P$ values, $t$-test false discovery rates (FDR), Wilcoxon $P$ values, and Wilcoxon FDRs. In case the MIRA peaks overlapped ( $+1 \mathrm{~kb})$ with the upstream, intragenic, or downstream region of known transcripts, these were annotated using information on strand $( \pm)$, start and end chromosomal coordinates, symbol, and accession number.

Additional file 9: MIRA-chip profile at the top hits from a key study reporting transgenerationally inherited DNA methylation aberrations. We treated prospermatogonia of G1 fetuses with VZ or oil control in utero as depicted in Additional file 4, and mapped DNA methylation using MIRA-chip and CpG-promoter Nimblegen arrays in purified G1R and G2R prospermatogonia (MGC) at $17.5 \mathrm{dpc}$ and in adult spermatozoa. DNA methylation signals of MIRA versus input DNA were plotted as $-\log 10$ $P$ values ranging from 0 to 8.3 for experimental and control replicate samples as indicated to the left. The regions represent the top hits from [43] where these regions exhibited the greatest decrease and increase (Mro and Elf3, respectively) in sperm of $\mathrm{G} 3$ adult males after in utero exposure of prospermatogonia inside G1 fetuses, and were considered examples for transgenerational epigenetic aberrations. Note the lack of change in GIR and G2R MGC and sperm at these locations (black triangles) and the complete lack of DNA methylation at the Elf3 promoter at all times.

Additional file 10: No transgenerational epigenetic aberrations are detectable at IAP elements after in utero ED exposure. Samples (groups 5 to 8) were analyzed from the MIRA-chip experiment conducted using the custom imprinting arrays (Additional file 7). The average MIRA/input log2 ratios ( $n=3$ for MGCs and $n=2$ for sperm) were measured along 1-kb-long flanking region to each IAP (column A), at the chromosomal locations indicated (columns B to D), where these flanks were unique sequences. Sheet 1 in the Excel file provides the complete dataset of the level 1 analysis. The average values of two conditions (average sample A versus average sample B) were compared along these regions to detect changes with the cutoff values of $\pm 5 \%$ and $P<0.05$ (Student's $t$-test). The MIRA/input log2 ratios of each individual sample are also provided together with standard deviation values of the Student's $t$-test. Sheet 2 provides the result of the analysis where common changes were detected between MGC-sperm or between G1R and G2R samples. Sheet two provides the summary of common hits. In case the MIRA peaks overlapped $( \pm 10 \mathrm{~kb})$ with the upstream, intragenic, or downstream region of known transcripts, these were annotated using information on strand $( \pm)$, start and end chromosomal coordinates, symbol, and accession number. Note that there were very few immediate and persistent changes. These were small, often occurred in the opposite direction between generations, and rarely mapped to known transcripts.

Additional file 11: Search for persistent epigenetic aberrations at IAP elements. (A) Change in G1R MGC that persists into G2R MGC after in utero VZ exposure. DNA methylation was measured along each unique 1-kb-long IAP-flanking region in the MIRA-chip samples as shown at the top. Selected IAP-flank regions where common changes were detected in MGC samples between G1R MGC and G2R MGC are shown with chromosomal coordinates. The criteria for selection was the following: the change between GIR VZ versus G1R oil and also G2R VZ and G2R oil were at least $\pm 5 \%$ with $P<0.05$ (Student's $t$-test). The average MIRA/input log2 ratios ( $n=3$ for MGC and $n=2$ for sperm) are depicted with red and blue flags in the range of -1.2 to +2.1 . In case the MIRA peaks overlapped $( \pm 10 \mathrm{~kb})$ with the upstream, intragenic, or downstream region of known transcripts, these were marked at the right using strand information $( \pm)$. (B) Search for epigenetic aberrations at IAP elements in GIR MGC that persist into GIR sperm after in utero VZ exposure. The criteria for selection were the following: the change between MGC G1R VZ versus G1R oil and also between sperm G1R VZ and G2R oil was at least $\pm 5 \%$ with $P<0.05$. (C) Search for epigenetic aberrations at IAP elements in G1R sperm that persist into G2R sperm after in utero VZ exposure. The criteria for selection was the following: the change between sperm GIR VZ versus G1R oil and also between sperm G2R VZ and G2R oil were at least $\pm 5 \%$ with $P<0.05$. (D) Search for epigenetic aberrations at IAP elements in G1R MGC that persist into G2R MGC after in utero DEHP exposure. The criteria for selection were the following: the change between G1R DEHP versus G1R oil and also G2R DEHP and G2R oil were at least $\pm 5 \%$ with $P<0.05$. (E) Search for epigenetic aberrations at IAP elements in GIR MGC that persist into G2 MGC after in utero BPA exposure. The criteria for selection were the following: the change between G1R BPA versus G1R oil and also G2R BPA and G2R oil were at least $\pm 5 \%$ with $P<0.05$. Note that there were very few immediate and persistent changes. These were small and often occurred in the opposite direction between generations (bottom group). The full set of data and calculations are provided in Additional file 10.

Additional file 12: Common changes of transcription in G1R and G2R prospermatogonia. (A) Common changes after DEHP treatment. (B) Common changes after VZ treatment. Full results of the common hits tabulated in Table 4 is shown. Common changes were detected between the unique transcripts that change in G1R and G2R samples for the same treatment using 1.05 -fold difference and $P<0.05$ cutoff values. We marked those that changed in the same direction.

Additional file 13: List of primers used for Sequenom allelotyping. Primers are listed for Sequenom experiments. The first PCR primer (1st-PCRP) and the second PCR primer (2nd-PCRP) were used in the amplification. UEP_SEQ primers were used in mass spectrometry after incorporating one or the other allele-specific nucleotide by primer extension. The DMR18-plex was used for DNA methylation analysis at DMR regions. The imprinted expression multiplexes were used for allele-specific transcription analysis at imprinted genes. 


\section{Abbreviations}

BPA: Bisphenol A; DEHP: Di-(2-ethylhexyl) phthalate; DMR: Differentially methylated region; ED: Endocrine-disrupting chemical; FDR: False discovery rate; FGC: Female germ cell; FSC: Female somatic cell; G1R: Generation 1-reprogrammed; G2R: Generation 2-reprogrammed; IAP: Intracisternal A-particle element; MAT: Maternally methylated; MGC: Male germ cell; MIRA: Methylated CpG island recovery assay; MSC: Male somatic cell; PAT: Paternally methylated; PCA: Principal component analysis; PGC: Primordial germ cells; TGl: Transgenerational inheritance; VZ: Vinclozolin.

\section{Competing interests}

The author(s) declare that they have no competing interests.

\section{Authors' contributions}

DAT and $\mathrm{KI}$ carried out the experiments with help from AYB and PS. AXL, CW, and XW provided microarray data analysis. GPP participated in the study design and manuscript revision. PES conceived and designed the study, analyzed the results, and wrote the manuscript. All authors read and approved the final manuscript.

\section{Acknowledgements}

We are grateful to the Animal Research Center of the City of Hope for providing animal care, and to Alexander Spalla and Lucy Brown (Analytical Cytometry Core, City of Hope) for FACS sorting of germ cells. We thank Ning Ye for performing the Affymetrix microarray sample labeling and hybridizations. We thank Xinmin Li, Jamie Zhou, and Jose Solis (UCLA Microarray Core) for Nimblegen array hybridizations. We thank Dr. Toshihiko Shiroishi, (National Institute of Genetics, Mishima, Japan) for the JF1/Ms inbred mouse line (JAX 003720). We thank David Nadziejka (Van Andel Institute) for technical editing of the revised manuscript and Cynthia Armstrong for helping with the Figures. This work was supported by grants RO1ES015185 and RO1GM064378 from the National Institute of Health to PES. The funders had no role in study design, data collection and analysis, decision to publish, or preparation of the manuscript.

\section{Author details}

'Department of Molecular and Cellular Biology, Beckman Research Institute, City of Hope, Duarte, California 91010, USA. ${ }^{2}$ Irell and Manella Graduate School of Biological Sciences, City of Hope, Duarte, California 91010, USA. ${ }^{3}$ Department of Information Science, Beckman Research Institute, City of Hope, Duarte, California 91010, USA. ${ }^{4}$ Eugene and Ruth Roberts Summer Academy, City of Hope, Duarte, California 91010, USA. ${ }^{5}$ Department of Cancer Biology, Beckman Research Institute, City of Hope, Duarte, California 91010, USA. ${ }^{6}$ Center for Epigenetics, Van Andel Research Institute, Grand Rapids, Michigan 49503, USA.

Received: 23 December 2014 Accepted: 23 February 2015 Published online: 27 March 2015

\section{References}

1. Calafat AM, Ye X, Wong LY, Reidy JA, Needham LL. Exposure of the U.S. population to bisphenol A and 4-tertiary-octylphenol: 2003-2004. Environ Health Perspect. 2008;116:39-44.

2. Mendiola J, Meeker JD, Jorgensen N, Andersson AM, Liu F, Calafat AM, et al. Urinary concentrations of di(2-ethylhexyl) phthalate metabolites and serum reproductive hormones: pooled analysis of fertile and infertile men. J Androl. 2012;33:488-98.

3. Sathyanarayana S, Calafat AM, Liu F, Swan SH. Maternal and infant urinary phthalate metabolite concentrations: are they related? Environ Res. 2008;108:413-8.

4. Vandenberg LN, Chahoud I, Heindel JJ, Padmanabhan V, Paumgartten FJ, Schoenfelder G. Urinary, circulating, and tissue biomonitoring studies indicate widespread exposure to bisphenol A. Environ Health Perspect. 2010;118:1055-70.

5. Wickerham EL, Lozoff B, Shao J, Kaciroti N, Xia Y, Meeker JD. Reduced birth weight in relation to pesticide mixtures detected in cord blood of full-term infants. Environ Int. 2012;47:80-5.

6. Anway MD, Cupp AS, Uzumcu M, Skinner MK. Epigenetic transgenerational actions of endocrine disruptors and male fertility. Science. 2005;308:1466-9.
7. Swan SH, Main KM, Liu F, Stewart SL, Kruse RL, Calafat AM, et al. Decrease in anogenital distance among male infants with prenatal phthalate exposure. Environ Health Perspect. 2005;113:1056-61.

8. Vom Saal FS, Cooke PS, Buchanan DL, Palanza P, Thayer KA, Nagel SC, et al. A physiologically based approach to the study of bisphenol $A$ and other estrogenic chemicals on the size of reproductive organs, daily sperm production, and behavior. Toxicol Ind Health. 1998;14:239-60.

9. Agarwal DK, Eustis S, Lamb JCT, Reel JR, Kluwe WM. Effects of di(2-ethylhexyl) phthalate on the gonadal pathophysiology, sperm morphology, and reproductive performance of male rats. Environ Health Perspect. 1986;65:343-50.

10. Anway MD, Memon MA, Uzumcu M, Skinner MK. Transgenerational effect of the endocrine disruptor vinclozolin on male spermatogenesis. J Androl. 2006:27:868-79.

11. Jirtle RL, Skinner MK. Environmental epigenomics and disease susceptibility. Nat Rev Genet. 2007;8:253-62.

12. Anway MD, Rekow SS, Skinner MK. Transgenerational epigenetic programming of the embryonic testis transcriptome. Genomics. 2008;91:30-40.

13. Manikkam M, Tracey R, Guerrero-Bosagna C, Skinner MK. Plastics derived endocrine disruptors (BPA, DEHP and DBP) induce epigenetic transgenerational inheritance of obesity, reproductive disease and sperm epimutations. PLoS One. 2013;8:e55387.

14. Skinner MK, Mohan M, Haque MM, Zhang B, Savenkova MI. Epigenetic transgenerational inheritance of somatic transcriptomes and epigenetic control regions. Genome Biol. 2012;13:R91.

15. Sasaki H, Matsui $Y$. Epigenetic events in mammalian germ-cell development: reprogramming and beyond. Nat Rev Genet. 2008;9:129-40.

16. Guibert S, Forne T, Weber M. Global profiling of DNA methylation erasure in mouse primordial germ cells. Genome Res. 2012;22:633-41.

17. Kobayashi H, Sakurai T, Miura F, Imai M, Mochiduki K, Yanagisawa E, et al. High-resolution DNA methylome analysis of primordial germ cells identifies gender-specific reprogramming in mice. Genome Res. 2013;23:616-27.

18. Seisenberger S, Andrews S, Krueger F, Arand J, Walter J, Santos F, et al. The dynamics of genome-wide DNA methylation reprogramming in mouse primordial germ cells. Mol Cell. 2012:48:849-62.

19. Singh P, Li AX, Tran DA, Oates N, Kang ER, Wu X, et al. De novo DNA methylation in the male germ line occurs by default but is excluded at sites of H3K4 methylation. Cell Rep. 2013;4:205-19.

20. Kobayashi H, Sakurai T, Imai M, Takahashi N, Fukuda A, Yayoi O, et al Contribution of intragenic DNA methylation in mouse gametic DNA methylomes to establish oocyte-specific heritable marks. PLoS Genet. 2012:8:e1002440

21. Smallwood SA, Tomizawa S, Krueger F, Ruf N, Carli N, Segonds-Pichon A, et al, Dynamic CpG island methylation landscape in oocytes and preimplantation embryos. Nat Genet. 2011;43:811-4.

22. Jirtle RL, Sander M, Barrett JC. Genomic imprinting and environmental disease susceptibility. Environ Health Perspect. 2000;108:271-8.

23. Lim AL, Ferguson-Smith AC. Genomic imprinting effects in a compromised in utero environment: implications for a healthy pregnancy. Semin Cell Dev Biol. 2010:21:201-8.

24. Grossniklaus U, Kelly B, Ferguson-Smith AC, Pembrey M, Lindquist S. Transgenerational epigenetic inheritance: how important is it? Nat Rev Genet. 2013;14:228-35.

25. Ferguson-Smith AC. Genomic imprinting: the emergence of an epigenetic paradigm. Nat Rev Genet. 2011;12:565-75.

26. Hajkova P, Erhardt S, Lane N, Haaf T, El-Maarri O, Reik W, et al. Epigenetic reprogramming in mouse primordial germ cells. Mech Dev. 2002;117:15-23.

27. Yamazaki Y, Low EW, Marikawa Y, Iwahashi K, Bartolomei MS, McCarrey JR, et al. Adult mice cloned from migrating primordial germ cells. Proc Natl Acad Sci U S A. 2005;102:11361-6.

28. Szabó PE, Hubner K, Scholer H, Mann JR. Allele-specific expression of imprinted genes in mouse migratory primordial germ cells. Mech Dev. 2002;115:157-60

29. Szabó PE, Mann JR. Biallelic expression of imprinted genes in the mouse germ line: implications for erasure, establishment, and mechanisms of genomic imprinting. Genes Dev. 1995;9:1857-68.

30. Kato Y, Kaneda M, Hata K, Kumaki K, Hisano M, Kohara Y, et al. Role of the Dnmt3 family in de novo methylation of imprinted and repetitive sequences during male germ cell development in the mouse. Hum Mol Genet. 2007;16:2272-80. 
31. Smith ZD, Chan MM, Mikkelsen TS, Gu H, Gnirke A, Regev A, et al. A unique regulatory phase of DNA methylation in the early mammalian embryo. Nature. 2012;484:339-44.

32. Kang ER, lqbal $K$, Tran DA, Rivas GE, Singh P, Pfeifer GP, et al. Effects of endocrine disruptors on imprinted gene expression in the mouse embryo. Epigenetics. 2011;6:7.

33. Zhang XF, Zhang LJ, Feng YN, Chen B, Feng YM, Liang GJ, et al. Bisphenol A exposure modifies DNA methylation of imprint genes in mouse fetal germ cells. Mol Biol Rep. 2012;39:8621-8.

34. Stouder C, Paoloni-Giacobino A. Transgenerational effects of the endocrine disruptor vinclozolin on the methylation pattern of imprinted genes in the mouse sperm. Reproduction. 2010;139:373-9.

35. Stouder C, Paoloni-Giacobino A. Specific transgenerational imprinting effects of the endocrine disruptor methoxychlor on male gametes. Reproduction. 2011;141:207-16

36. Chao HH, Zhang XF, Chen B, Pan B, Zhang LJ, Li L, et al. Bisphenol A exposure modifies methylation of imprinted genes in mouse oocytes via the estrogen receptor signaling pathway. Histochem Cell Biol. 2012;137:249-59.

37. Susiarjo M, Sasson I, Mesaros C, Bartolomei MS. Bisphenol a exposure disrupts genomic imprinting in the mouse. PLoS Genet. 2013;9:e1003401.

38. Marques CJ, Carvalho F, Sousa M, Barros A. Genomic imprinting in disruptive spermatogenesis. Lancet. 2004;363:1700-2.

39. Marques CJ, Francisco T, Sousa S, Carvalho F, Barros A, Sousa M. Methylation defects of imprinted genes in human testicular spermatozoa. Fertil Steril. 2010;94:585-94.

40. Rauch TA, Pfeifer GP. The MIRA method for DNA methylation analysis. Methods Mol Biol. 2009;507:65-75.

41. Lee DH, Tran DA, Singh P, Oates N, Rivas GE, Larson GP, et al. MIRA-SNuPE, a quantitative, multiplex method for measuring allele-specific DNA methylation. Epigenetics. 2011;6:212-23.

42. Singh P, Wu X, Lee DH, Li AX, Rauch TA, Pfeifer GP, et al. Chromosome-wide analysis of parental allele-specific chromatin and DNA methylation. Mol Cell Biol. 2011;31:1757-70.

43. Guerrero-Bosagna C, Covert TR, Haque MM, Settles M, Nilsson EE, Anway $M D$, et al. Epigenetic transgenerational inheritance of vinclozolin induced mouse adult onset disease and associated sperm epigenome biomarkers. Reprod Toxicol. 2012;34:694-707.

44. Tickner JA, Schettler T, Guidotti T, McCally M, Rossi M. Health risks posed by use of Di-2-ethylhexyl phthalate (DEHP) in PVC medical devices: a critical review. Am J Ind Med. 2001;39:100-11.

45. Kimble J, Hirsh D. The postembryonic cell lineages of the hermaphrodite and male gonads in Caenorhabditis elegans. Dev Biol. 1979;70:396-417.

46. Kelly WG. Transgenerational epigenetics in the germline cycle of Caenorhabditis elegans. Epigenetics Chromatin. 2014;7:6.

47. Greer EL, Maures TJ, Ucar D, Hauswirth AG, Mancini E, Lim JP, et al. Transgenerational epigenetic inheritance of longevity in Caenorhabditis elegans. Nature. 2011;479:365-71.

48. Moore RW, Rudy TA, Lin TM, Ko K, Peterson RE. Abnormalities of sexual development in male rats with in utero and lactational exposure to the antiandrogenic plasticizer Di(2-ethylhexyl) phthalate. Environ Health Perspect. 2001;109:229-37.

49. Timms BG, Howdeshell KL, Barton L, Bradley S, Richter CA, Vom Saal FS. Estrogenic chemicals in plastic and oral contraceptives disrupt development of the fetal mouse prostate and urethra. Proc Natl Acad Sci U S A. 2005;102:7014-9.

50. Buckley J, Willingham E, Agras K, Baskin LS. Embryonic exposure to the fungicide vinclozolin causes virilization of females and alteration of progesterone receptor expression in vivo: an experimental study in mice. Environ Health. 2006;5:4.

51. Vilela ML, Willingham E, Buckley J, Liu BC, Agras K, Shiroyanagi Y, et al. Endocrine disruptors and hypospadias: role of genistein and the fungicide vinclozolin. Urology. 2007;70:618-21.

52. Elzeinova F, Novakova V, Buckiova D, Kubatova A, Peknicova J. Effect of low dose of vinclozolin on reproductive tract development and sperm parameters in CD1 outbred mice. Reprod Toxicol. 2008;26:231-8.

53. Lamb JC, Chapin RE, Teague J, Lawton AD, Reel JR. Reproductive effects of four phthalic acid esters in the mouse. Toxicol Appl Pharmacol. 1987;88:255-69.
54. Hayashi $Y$, Ito $Y$, Yanagiba $Y$, Kamijima M, Naito H, Nakajima T. Differences in metabolite burden of di(2-ethylhexyl)phthalate in pregnant and postpartum dams and their offspring in relation to drug-metabolizing enzymes in mice. Arch Toxicol. 2012;86:563-9.

55. Kawamoto Y, Matsuyama W, Wada M, Hishikawa J, Chan MP, Nakayama A, et al. Development of a physiologically based pharmacokinetic model for bisphenol A in pregnant mice. Toxicol Appl Pharmacol. 2007;224:182-91.

56. Susiarjo M, Hassold TJ, Freeman E, Hunt PA. Bisphenol A exposure in utero disrupts early oogenesis in the mouse. PLoS Genet. 2007;3:e5.

57. Lawson C, Gieske M, Murdoch B, Ye P, Li Y, Hassold T, et al. Gene expression in the fetal mouse ovary is altered by exposure to low doses of bisphenol $A$. Biol Reprod. 2011;84:79-86.

58. Lee DH, Singh P, Tsai SY, Oates N, Spalla A, Spalla C, et al. CTCF-dependent chromatin bias constitutes transient epigenetic memory of the mother at the H19-lgf2 imprinting control region in prospermatogonia. PLoS Genet. 2010;6:e1001224

59. Rauch TA, Pfeifer GP. DNA methylation profiling using the methylated-CpG island recovery assay (MIRA). Methods. 2010;52:213-7.

60. Irizarry RA, Hobbs B, Collin F, Beazer-Barclay YD, Antonellis KJ, Scherf U, et al. Exploration, normalization, and summaries of high density oligonucleotide array probe level data. Biostatistics. 2003;4:249-64.

61. Szabó PE, Tang SH, Reed MR, Silva FJ, Tsark WM, Mann JR. The chicken beta-globin insulator element conveys chromatin boundary activity but not imprinting at the mouse lgf2/H19 domain. Development. 2002;129:897-904.

62. Singh P, Cho J, Tsai SY, Rivas GE, Larson GP, Szabó PE. Coordinated allele-specific histone acetylation at the differentially methylated regions of imprinted genes. Nucleic Acids Res. 2010;38:7974-90.

63. Singh $P$, Han L, Rivas GE, Lee $D H$, Nicholson TB, Larson GP, et al. Allele-specific H3K79 Di- versus trimethylation distinguishes opposite parental alleles at imprinted regions. Mol Cell Biol. 2010;30:2693-707.

\section{Submit your next manuscript to BioMed Central and take full advantage of:}

- Convenient online submission

- Thorough peer review

- No space constraints or color figure charges

- Immediate publication on acceptance

- Inclusion in PubMed, CAS, Scopus and Google Scholar

- Research which is freely available for redistribution 